\title{
Oxidative stress response pathways: Fission yeast as archetype
}

\section{Papadakis, Manos A.; Workman, Christopher}

\section{Published in:}

Critical Reviews in Microbiology

Link to article, DOI:

10.3109/1040841X.2013.870968

Publication date:

2015

Document Version

Publisher's PDF, also known as Version of record

Link back to DTU Orbit

\section{Citation (APA):}

Papadakis, M. A., \& Workman, C. (2015). Oxidative stress response pathways: Fission yeast as archetype. Critical Reviews in Microbiology, 41(4), 520-535. https://doi.org/10.3109/1040841X.2013.870968

\section{General rights}

Copyright and moral rights for the publications made accessible in the public portal are retained by the authors and/or other copyright owners and it is a condition of accessing publications that users recognise and abide by the legal requirements associated with these rights.

- Users may download and print one copy of any publication from the public portal for the purpose of private study or research.

- You may not further distribute the material or use it for any profit-making activity or commercial gain

- You may freely distribute the URL identifying the publication in the public portal

If you believe that this document breaches copyright please contact us providing details, and we will remove access to the work immediately and investigate your claim. 


\title{
Oxidative stress response pathways: Fission yeast as archetype
}

\author{
Manos A. Papadakis and Christopher T. Workman \\ Center for Biological Sequence Analysis, Department of Systems Biology, Technical University of Denmark, Lyngby, Denmark
}

\begin{abstract}
Schizosaccharomyces pombe is a popular model eukaryotic organism to study diverse aspects of mammalian biology, including responses to cellular stress triggered by redox imbalances within its compartments. The review considers the current knowledge on the signaling pathways that govern the transcriptional response of fission yeast cells to elevated levels of hydrogen peroxide. Particular attention is paid to the mechanisms that yeast cells employ to promote cell survival in conditions of intermediate and acute oxidative stress. The role of the Sty $1 / \mathrm{Spc} 1 / \mathrm{Phh} 1$ mitogen-activated protein kinase in regulating gene expression at multiple levels is discussed in detail.
\end{abstract}

Keywords

Adaptation, gene regulation, hydrogen peroxide, redox signaling, survival

\author{
History \\ Received 5 August 2013 \\ Revised 22 November 2013 \\ Accepted 27 November 2013 \\ Published online 24 February 2014
}

\section{Introduction}

All organisms are continuously exposed to environmental changes during their lifetime. Unicellular organisms are particularly susceptible to sudden fluctuations in their growth environment, including alterations in nutrients, temperature, $\mathrm{pH}$ and osmolarity. Eukaryotic cells are often exposed to reactive oxygen species (ROS) during the course of normal aerobic metabolism. Increased ROS levels can also challenge cells exposed to oxidizing agents, such as hydrogen peroxide (HP). An excess of ROS leads to oxidative stress by directly or indirectly damaging DNA, proteins and lipids (Toledano, 2003). On the other hand, there is evidence for the involvement of ROS in vital signaling functions associated with diverse cellular processes (Rhee, 2006; Veal et al., 2007).

Cells need therefore to precisely tune cellular ROS levels and employ oxidative stress defense mechanisms to maintain ROS homeostasis. Eukaryotic cells have indeed evolved sophisticated ways to sense and respond to ROS (Temple et al., 2005). Evolutionary conserved signal transducing proteins, namely mitogen-activated protein kinases (MAPKs), constitute a large family of proteins that connect cell-surface receptors to critical regulatory targets within the cells, thereby controlling cell survival and adaptation in response to mitogens and other stimuli (English et al., 1999). Furthermore, there exists a conserved subfamily of MAPKs, namely the stress-activated protein kinase (SAPK) subfamily, which responds to diverse stress types rather than mitogenic stimuli (Waskiewicz \& Cooper, 1995).

Address for correspondence: Manos Aristotle Papadakis, Department of Systems Biology, Technical University of Denmark (DTU), Kemitorvet, Building 208, Kgs. Lyngby 2800, Denmark. E-mail: papadakismns@gmail.com
In humans, ROS are implicated in aging, cancer, atherosclerosis, Alzheimer's and Parkinson's disease among others (Finkel \& Holbrook, 2000). Therefore, studies investigating the stress-mediated gene expression changes and the cellular mechanisms employed in response to oxidative stress have markedly increased in recent years, especially using model organisms.

The fission yeast Schizosaccharomyces pombe is a genetically amenable system that has limited genome redundancy, which makes it well suited for phenotypic studies and the investigation of stress responses in particular. Schizosaccharomyces pombe employs distinct molecular pathways to respond to stress triggered by different HP dosages. The complexity and high sophistication of these pathways are highly conserved in mammals, and the fission yeast SAPK pathway activates orthologous transcription factors in humans. Importantly, additional fission yeast regulators, such as two-component signaling proteins, are highly conserved in many fungal species. Fission yeast is thus often the model organism of choice for oxidative stress studies, which aim to understand how fungal pathogens respond to ROS and reveal the gene regulation mechanisms that govern human disease progression.

Several gene expression studies have uncovered the global transcriptional program of fission yeast cells in response to oxidative stimuli. Thus, a solid basis of stress response data is available for this yeast. The study of fission yeast responses to oxidative stress is currently a highly active field of research that contributes substantially to our understanding of how eukaryotic cells respond to intracellular redox imbalances.

The aim of the present review is to summarize the upto-date knowledge on the gene expression changes initiated in the model eukaryote $S$. pombe in response to oxidative stimuli. Moreover, a major goal of the review is to describe the substantial body of work that has been performed to reveal 
the molecular pathways that fission yeast employs in response to oxidative stimuli as well as the mechanisms by which gene expression changes are implemented.

To this direction we discuss the multi-level regulation of gene expression mediated by the Sty1 SAPK (also known as Spc1 or Phh1). Sty1 not only controls several aspects of normal fission yeast physiology (Sansó et al., 2011a), but it also plays a vital role in cell survival under diverse stress conditions, including oxidative, osmotic, heat and metal stress (Nguyen et al., 2000; Shiozaki \& Russell, 1995b). Sty1 exerts its effects on gene expression mainly through the phosphorylation and stabilization of the Atf1 transcription factor (also known as Gad7, Mts1 or Sss1), which is similar to the human ATF2 and binds closely related DNA sequences (Shiozaki \& Russell, 1996; Wilkinson et al., 1996). Hence, a large fraction of genes that are transcriptionally regulated through the Sty1Atf1 pathway in fission yeast are closely related to the ones regulated via orthologous SAPKs in mammals. A number of additional molecular mechanisms are employed by Sty1 to regulate gene expression at the post-transcriptional level, interfere with the operation of the translational machinery and control the progression through the cell cycle. All these mechanisms are discussed in detail in the following sections.

\section{Stress responses cause widespread changes in transcription}

Cellular adaptation and survival in changing environments requires the rapid reprogramming of the transcriptional machinery towards molecular damage repair and cell survival (Estruch, 2000). The degree of stress resistance is inversely correlated with the rate of cellular growth. Therefore, upon stress unicellular organisms are challenged to balance two antagonistic gene expression programs, the growth- and the stress-related programs (López-Maury et al., 2008). Indeed, hundreds of growth-related genes in the budding yeast Saccharomyces cerevisiae show a considerable overlap with core stress genes in a way that the latter are expressed at low levels in normal growth and vice versa. Thus, many stress genes do not respond to stress, but to a reduction in growth rate caused by stress (Brauer et al., 2008; Castrillo et al., 2007).

Genome-wide expression profiling in model organisms, including the budding and fission yeasts, has provided insight into major principles of eukaryotic transcriptional regulation in response to a wide range of stress conditions (Chen et al., 2003; Gasch et al., 2000). These studies identified hundreds of genes with significantly altered transcript levels in stress that account for $10-15 \%$ of all protein-coding genes in the genome (Chen et al., 2003; Gasch et al., 2000). In both yeasts the magnitude and the duration of a stress response is proportionally affected by the dose of the stressor, with each stress condition resulting in a distinct expression signature (López-Maury et al., 2008).

Transcriptional responses are transient and mRNA levels reach, after some time, a new steady state that is close to the one prior to stress (Chen et al., 2008; Hughes et al., 2000). It has been recently shown that the treatment of $S$. cerevisiae cells with cumene hydroperoxide results in the transient induction and repression of approximately hundred genes within 6 minutes of exposure to the oxidizing agent
(Sha et al., 2013). Another study in budding yeast revealed that the response to the fungicide benomyl involves the alteration of transcript levels already 30 seconds after the addition of the drug (Lucau-Danila et al., 2005). Moreover, a recent study in $S$. pombe has shown that both the mRNA- and protein-level responses to heat and oxidative stress are rapid, with the former peaking at 15 minutes and the latter at 60 minutes after stress induction (Lackner et al., 2012).

Schizosaccharomyces pombe and S. cerevisiae launch transcriptional programs that are commonly induced in most of the stress conditions that have been examined. The core transcriptional response to diverse stress conditions is known as the environmental stress response (ESR) in S. cerevisiae (Gasch et al., 2000) and the core environmental stress response (CESR) in S. pombe (Chen et al., 2003). Notably, ESR and CESR are largely conserved between the two evolutionary distant yeasts (Causton et al., 2001; Chen et al., 2003; Gasch et al., 2000). The phenomenon of crossprotection, whereby cells treated with mild concentrations of a stressor become more resistant upon exposure to other stress agents, could be explained by such general-purpose responses (Kültz, 2005).

In fission yeast, 140 and 106 CESR genes are, respectively, induced and repressed ( $>2$-fold change) in most of the five stress conditions that have been tested, i.e. heat, osmotic, oxidative, heavy metal and DNA-damage stress (Chen et al., 2003). Genes with very different functions are commonly induced, while growth-related and energy consumption processes are often negatively regulated (Figure 1). Less conservative lists that include hundreds of CESR genes from each regulated group have also been reported. Genes in these lists are consistently regulated in response to most stress conditions but marginally fail the selection criterion ( $>2$-fold change) in some of the conditions (Chen et al., 2003).

The regulation of CESR genes is largely dependent on the Sty1 MAPK. However, the Atf1-binding site, i.e. cAMPresponsive element (CRE), is over-represented only in the promoter regions of induced CESR genes, while the repressed CESR group is virtually independent of Atf1 (Chen et al., 2003).

Furthermore, fission yeast extensively redirects its gene expression program in response to different doses of HP, with the expression of 1800-3000 genes changing significantly in at least one HP concentration $(0.07,0.5$ or $6.0 \mathrm{mM})$ and one time point, respectively. Cellular responses are similar upon exposure to medium $(0.5 \mathrm{mM})$ or very high HP concentrations $(6.0 \mathrm{mM})$, while the response to low HP doses $(0.07 \mathrm{mM})$ is weak and triggers expression changes to only a few genes (Chen et al., 2008). A group of 127 core oxidative stress genes (COSG) (Chen et al., 2008) is induced in stress caused by a wide range of HP concentrations $(0.07,0.5$ and $6.0 \mathrm{mM})$. COSG are also hyper-induced in stress imposed by two other oxidizing agents, namely tert-butyl hydroperoxide and menadione (Chen et al., 2008).

Transcriptional regulation in HP stress is conserved across different kingdoms for a group of four protein families. This small group constitutes the so-called "core eukaryotic HP response" and includes G proteins, ubiquitin-conjugating enzymes, heat shock proteins and calcium-dependent protein kinases (Vandenbroucke et al., 2008). The conserved 
Carbohydrate metabolism Lipid or fatty acid metabolism Signaling and transcriptional regulation Antioxidants

DNA repair

Protein folding and degradation Transporters

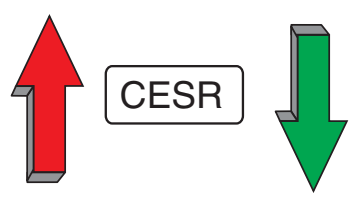

Transcription

Translation initiation

RNA processing and splicing

Ribosome biogenesis

Transport

Signaling

Cytoskeletal organization

Figure 1. Biological processes affected by diverse environmental perturbations. Arrows pointing up- and downwards are used to indicate gene induction and repression respectively. CESR, core environmental stress response.

(A)

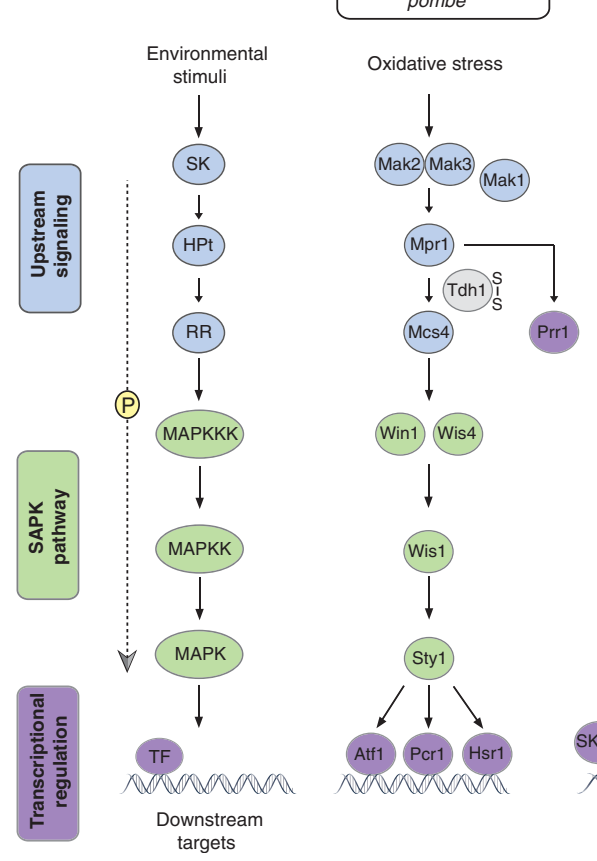

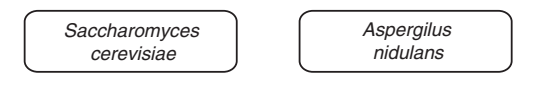

Osmotic stress

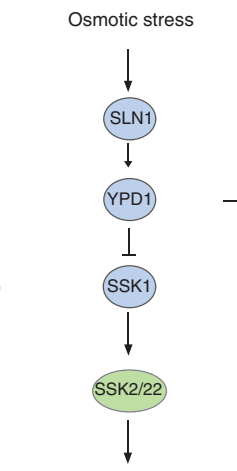

Oxidative and osmotic stress
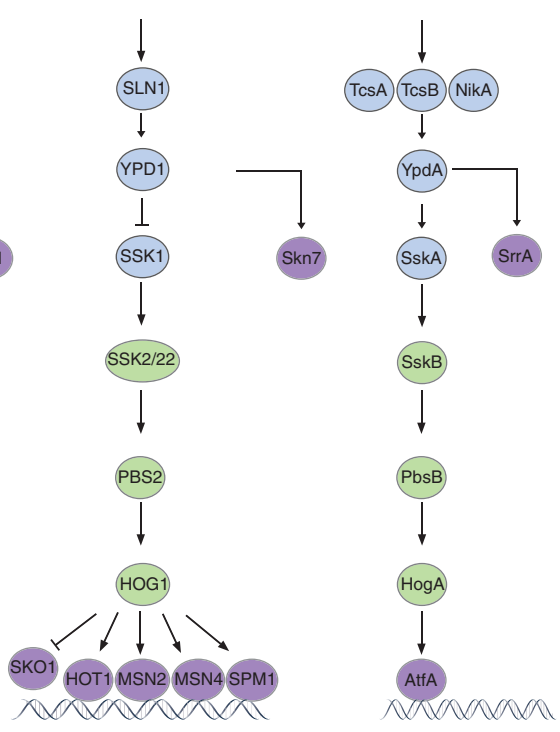
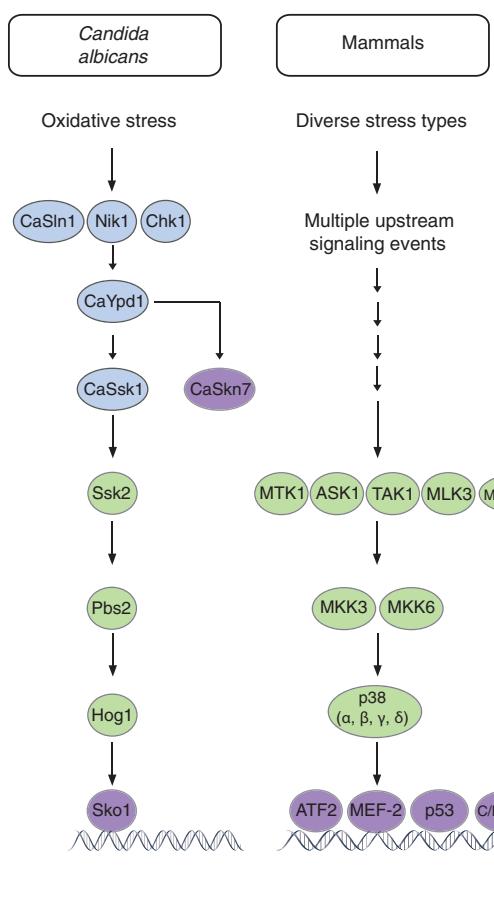
Multiple upstream
signaling events
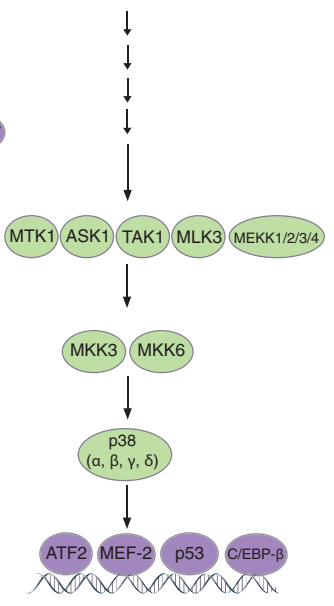

Figure 2. Upstream stress-sensing mechanisms and the mitogen-activated protein kinase (MAPK) family of proteins form a complex molecular network in response to environmental stimuli. (A) The basic architecture of MAPK pathways. TF, transcription factor; MAPKKK, mitogen-activated protein kinase kinase kinase; MAPKK, mitogen-activated protein kinase kinase; MAPK, mitogen-activated protein kinase. (B) A comparative view of stress sensing and signaling mechanisms in fungi and mammals reveals highly conserved modules.

inductive transcriptional response to HP in yeasts $(S$. pombe and $S$. cerevisae) (Chen et al., 2003) is more conserved in plants than in animals (Vandenbroucke et al., 2008). Interestingly, the increased expression of antioxidant enzymes being caused by elevated HP levels is not always observed for cells of multicellular organisms (Desaint et al., 2004), but it appears to be a universal response in unicellular organisms (Vandenbroucke et al., 2008).

\section{Stress signaling pathways: sensing of stress through complex upstream events}

The ability of an organism to constantly sense and respond to stimuli (or signals) involves extensive interactions between the environment and the genome. In challenging conditions a complex network of pathways and molecular interactions mediates genome-environment interactions to promote cell survival. Signals are processed not only via linear kinase cascades but also through networks of cross-talking pathways involving TFs, protein kinases (PKs), RNA-binding proteins (RBPs), small interfering RNAs and other biomolecules. Signals not only alter the transcriptional activity of the cell, but also affect mRNA half-lives, translation, protein half-lives, protein localization, protein activation and protein turnover.

Fungi and plants possess multistep phosphorelay systems, which are reminiscent of the bacterial two-component systems that transduce environmental signals to MAPKs, which in turn direct the reprogramming of the transcriptional machinery (Imamura et al., 1998; Stock et al., 2000). Unlike prokaryotic systems, where a stress signal is transduced from a histidine (His) kinase (sensor kinase) directly to a response regulator (RR), a third component exists in eukaryotic phosphorelay systems. This component is a His-containing phosphotransfer protein (HPt), which mediates the phosphotransfer between the sensor kinase and the RR (upper part of Figure 2A).

For instance, $S$. cerevisiae relays osmotic stress signals to the HOG1 MAPK pathway through the SLN1-YPD1-SSK1 phosphorelay system (Maeda et al., 1994; Posas et al., 1996), while the TcsA/TcsB/NikA sensor kinases transmit stress signals to the HogA/SakA MAPK cascade through YpdA and SskA in Aspergillus nidulans (Furukawa et al., 2005; Virginia et al., 2000). In Candida albicans, a similar system consists of the histidine kinases CaSln1, Chk1 and Nik1/Cos1 (Alex et al., 1998; Calera \& Calderone, 1999a; 
Nagahashi et al., 1998), the phosphorelay protein CaYpd1 and two RRs (CaSsk1, CaSkn7) (Calera \& Calderone, 1999b; Calera et al., 2000) (Figure 2B).

Schizosaccharomyces pombe possesses a similar multistep phosphorelay system (Figure 2B), which specifically senses and transduces oxidative stress signals through two highly related His kinases (Mak2/Phk1 and Mak3/Phk2), the Mpr1/ Spy1 HPt protein and the Mcs4 aspartic acid (Asp)-containing RR (Buck et al., 2001; Nguyen et al., 2000). Fission yeast possesses a second RR, namely Prr1 (Ohmiya et al., 1999; Shieh et al., 1997a), which plays an important role for cell viability in oxidative stress of different intensity (see following sections). Notably, a phosphorelay system similar to those identified in fungal species has not been identified in mammals.

\section{A closer look into the fission yeast phosphorelay system}

The fission yeast phosphorelay system operates by sequential phosphorylation of the component proteins, and it is dedicated to sense oxidative stress signals (Buck et al., 2001; Nguyen et al., 2000). Similar to prokaryotic organisms, the His sensor kinase (Mak2) could be potentially autophosphorylated in the expense of ATP upon intermediate and acute HP stress (Stock et al., 2000), but the validity of this regulatory event remains elusive. The phosphoryl group is sequentially transferred from Mak2 to downstream components (Mak3, Mpr1 and Mcs4) through the His-Asp phosphotransfer reaction (Figures 4 and 6) (Buck et al., 2001; Nguyen et al., 2000). The role of another His kinase, Mak1/Phk3, is only partially understood. At low HP concentrations Mak1 is possibly involved in the Pap1/Caf3- (see following sections) and/or Prr1-dependent transcription (Buck et al., 2001) and presumably reduces the extent of Sty1 activation by inhibiting Mpr1 and thus weakening the Mak2Mak3 signal (Figure 6) (Quinn et al., 2002).

The different arrangement of redox sensing domains in Mak1 compared with those in Mak2 and Mak3 is related to the differential regulation of the corresponding kinase domains upon stress (Buck et al., 2001). In HP stress two conserved protein domains in the structures of Mak2 and Mak3, i.e. the GAF and PAS domains, are essential for sensing and relaying signals to the downstream MAPK pathway (Quinn et al., 2011; Taylor \& Zhulin, 1999). Both domains bind diverse cofactors to sense different stimuli, with the PAS domain being also involved in protein-protein interactions (Erbel et al., 2003). Interestingly, 18 TFs have been identified as potential interaction partners of the Mak1-3 proteins, with Mak2 alone being predicted to physically interact with six TFs (Atf31, Hsr1, Rsv1, Res1, Moc3 and Prr1) (Pancaldi et al., 2012).

In addition to structural kinase domains, signal transduction through the phosphorelay system is facilitated by an unexpected mechanism. Upon exposure to very high HP concentrations $(>1.0 \mathrm{mM})$, the glycolytic enzyme glyceraldehyde-3-phosphate dehydrogenase (GAPDH) encoded by $t d h 1 /$ gpdl is transiently oxidized through S-thiolation of a conserved cysteine residue in the catalytic center (Cys-152). This molecular event renders the GAPDH enzyme inactive and ceases glycolysis (Grant et al., 1999; Morigasaki et al., 2008). The transient oxidation of Tdh1 enhances its interaction with Mcs4 and, therefore, facilitates the interaction between Mcs4 and Mpr1 (Figure 5). Though to a lesser extent, a second GAPDH (Gpd3) can also promote stress signaling through the phosphorelay system (Morigasaki et al., 2008).

In many species proteins involved in the transduction of environmental signals localize at the plasma membrane (Moskvina et al., 1999; Tatebayashi et al., 2007). Instead, the fission yeast His kinases are cytoplasmic and, thus, evolutionarily optimized to sense internal and external oxidative stress signals (Quinn et al., 2011). Mcs4 localizes in the cytoplasm, Mpr1 is located in both the cytoplasm and the nucleus and, the Prr1 RR resides constitutively in the nucleus (Quinn et al., 2011). Interestingly, this localization pattern is not altered after treatment of cells with HP (Figures 4-6) (Quinn et al., 2011). The nuclear localization of Mpr1 and Prr1 and the essential role of a conserved Prr1 residue (Asp-418) in stress induced by extreme HP doses $(>1.0 \mathrm{mM})$ led to the conclusion that Prr1 could be phosphorylated by Mpr1 to control its TF activity. However, the Mpr1-mediated phosphotransfer does not seem to affect Prr1 stabilization (Quinn et al., 2011).

\section{Stress signaling pathways: the central role of the MAPK family}

In many species the MAPK family of proteins plays a central role in mediating changes associated with perturbations in the microenvironment of the cell (Marshall, 1994). The basic architecture of a MAPK pathway consists of three sequentially acting PKs: MAPK kinase kinases (MAPKKKs) phosphorylate and activate MAPK kinases (MAPKKs), which in turn phosphorylate and activate MAPKs (lower part of Figure 2A) (Marshall, 1994; Waskiewicz \& Cooper, 1995).

SAPKs are members of a conserved subfamily of MAPKs that respond to diverse stress rather than mitogen stimuli (Waskiewicz \& Cooper, 1995). SAPK pathways are present in all eukaryotic organisms and possess an essential role in the adaptation and survival of the cell in different hostile environments. Similar to MAPKs, SAPKs are activated through a series of upstream events to induce the expression of stress-protective genes and coordinate diverse cellular processes that confer stress resistance (Kyriakis \& Avruch, 2001). The SAPK modules that are present in fungal species are remarkably conserved in mammals (Figure 2B): Win1/ Wis4-Wis1-Sty1 (S. pombe) (Millar et al., 1995; Samejima et al., 1997; Shieh et al., 1997a), SSK2/22-PBS2-HOG1 (S. cerevisiae) (Brewster et al., 1993; Posas \& Saito, 1998; Posas et al., 1996), SskB-PbsB-HogA (A. nidulans) (Aguirre et al., 2005; Kawasaki et al., 2002; Vargas-Pérez et al., 2007), Ssk2-Pbs2-Hog1 (C. albicans) (Cheetham et al., 2007; San José et al., 1996) and MTK1/ASK1/TAK1/MLKs/MEKKsMKKs-p38 $(\alpha, \beta, \gamma, \delta)$ (mammals) (Mielke \& Herdegen, 2000; Nebreda \& Porras, 2000; Paul et al., 1997; Tibbles \& Woodgett, 1999). Among other TFs, the mammalian p38 SAPK pathway regulates ATF2, which is the ortholog of the fission yeast Atf1 TF (Table 1 and Figure 2B) (Dérijard et al., 1994). 


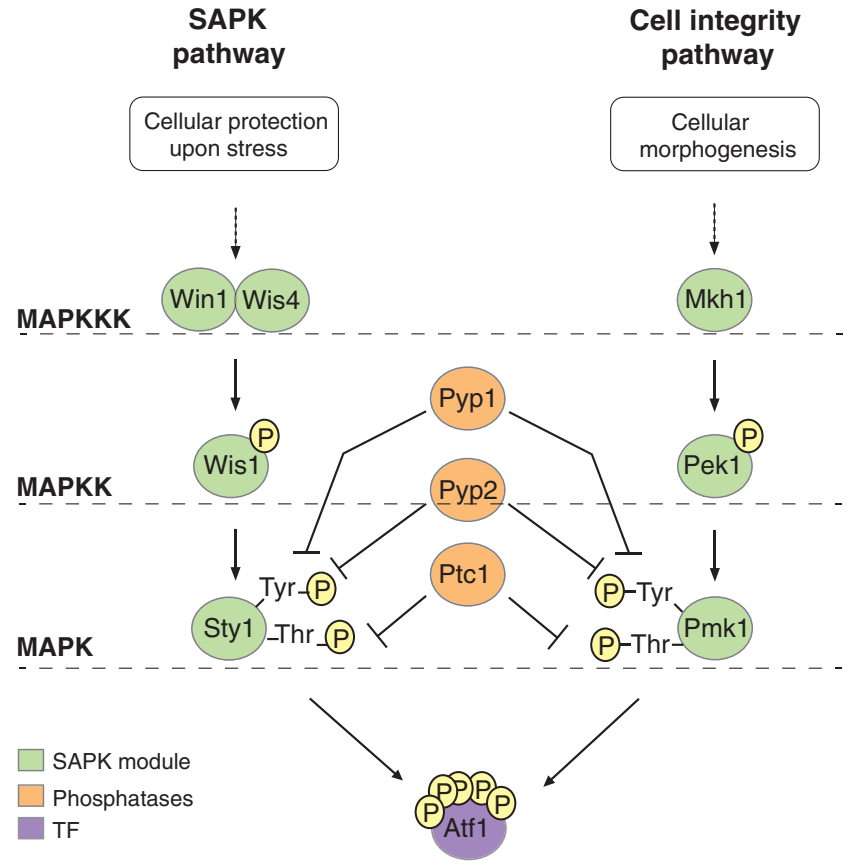

Figure 3. Molecular and functional overlap of the fission yeast stressactivated protein kinase (SAPK) and the cell integrity pathways. Colors used to represent different type of molecules are explained in the lower left part of the figure.

In $S$. pombe, three distinct MAPK pathways have been identified to date: the cell integrity (Pmk1/Spm1) (Toda et al., 1996), pheromone signaling (Spk1) (Toda et al., 1991) and SAPK (Sty1) pathways (Millar et al., 1995; Shiozaki \& Russell, 1995a). As opposed to six or more MAPK pathways in S. cerevisiae (Hohmann, 2002), the fission yeast MAPK pathways are tightly connected with each other. For instance, the Pmk1 and Sty1 MAPKs not only share regulatory links (i.e. the Pyp1, Pyp2 and Ptc1 protein phosphatases) (Takada et al., 2007), but also target the same TF (i.e. Atf1) to regulate gene expression through the cell integrity and stress response pathway respectively (Figure 3). Furthermore, a considerable functional overlap between the two pathways exists, with their role in controlling cellular morphogenesis being equally important (Madrid et al., 2007). Nevertheless, cellular protection against environmental insults depends heavily on the Sty1 pathway, as demonstrated by sensitivity tests with relevant deletion mutants (Pérez \& Cansado, 2010). Specifically, activation of the Sty1 SAPK in fission yeast is essential to promote cell survival in osmotic, oxidative, heat, heavy metal and nutrient stress (Degols \& Russell, 1997; Nguyen et al., 2002; Samejima et al., 1997; Shieh et al., 1997b; Shiozaki \& Russell, 1995b).

Although the stress signal is most often relayed to the Sty1 MAPK through MAPKKKs (Ikner \& Shiozaki, 2005), there exists evidence for the activation of Sty1 in a MAPKKKindependent manner. For instance, stress imposed by cadmium or low glucose concentrations bypasses MAPKKKs and stimulates the Sty1 pathway through the oxidation-dependent inhibition of the Pyp1 phosphatase (Zhou et al., 2010), which deactivates Sty1 in normal conditions of growth (Shiozaki \& Russell, 1995a). Similarly, upon heat stress Sty1 becomes activated after the abolishment of the binding between Sty1 and Pyp1 (Nguyen \& Shiozaki, 1999). However, osmotic and oxidative stress signals from the phosphorelay system are relayed to downstream proteins of the fission yeast MAPK pathway in a MAPKKK-dependent manner (see next section) (Buck et al., 2001; Ikner \& Shiozaki, 2005).

\section{Activation of Sty1 results in redirection of gene expression to promote cell survival in oxidative stress}

At the intersection of the phosphorelay system with the SAPK pathway, Mcs4 binds and activates the Wis4/Wak1/Wik1 and Win1 MAPKKKs (Shieh et al., 1997b), which are functionally redundant in phosphorylating the Wis1/Spc2/Smf2 MAPKK (Degols \& Russell, 1997). In turn, activated Wis1 phosphorylates the Sty1 MAPK, which becomes fully activated by the dual phosphorylation of a threonine and a tyrosine residue (Thr-171 and Tyr-173) in a Thr-XXX-Tyr motif within the activation loop (Figure 3) (Robbins et al., 1993). Notably, at very high HP dosages $(>1.0 \mathrm{mM})$ the noncatalytic domains of Wis4 and Win 1 interact constitutively with the Tdh1 GAPDH, but the interaction is not required for the association of Mcs4 with the MAPKKKs (Morigasaki et al., 2008). Wis1 not only phosphorylates but also targets Sty1 to the nucleus by the use of a MAPK-docking site and a nuclear export signal, which are present in the structure of the protein (Figure 4) (Nguyen et al., 2002).

Specifically upon oxidative stress and after Wis1-mediated phosphorylation of Sty1, the thioredoxin peroxidase Tpx1 activates the MAPK by a distinct mechanism, which does not involve the peroxidase activity of Tpx1 but affects the redox state of the latter. In particular, HP induces the formation of a transient disulphide complex between Tpx1 (Cys-48) and Sty1 (Cys-35) (Figure 4) (Veal et al., 2004). Additional redoxsensitive cysteine(s) present in the Sty 1 protein or the reduced form of the thioredoxin Trx 1 could further control the activation of Sty1 downstream of the SAPK pathway (Day et al., 2012; Veal et al., 2004).

After dual phosphorylation and Tpx1-mediated oxidation in the cytoplasm, activated Sty 1 translocates in the nucleus (Gaits et al., 1998), where it phosphorylates the Atf1 TF (Wilkinson et al., 1996). Although Sty1-mediated phosphorylation of Atf1 is required for the activation of its TF activity (Sansó et al., 2008), this phosphorylation event mainly promotes the stability of the Atf1 protein (Lawrence et al., 2007). Notably, there are 11 MAPK phosphorylation sites in Atf1 and the pattern of phosphorylation varies with both the time and type of stress (Quinn et al., 2002). Hyperphosphorylation and stabilization of Atf1 disrupts the preexisting association of the protein with a Skp, Cullin, F-box containing (SCF) E3 ligase, Fbh1/Fdh/Fdh1 (Lawrence et al., 2009). Upon exposure to intermediate HP levels $(0.25-1.0 \mathrm{mM})$ the constitutive recycling of Atf1 through the ubiquitin-proteasome system is in this manner prevented, a fact that results in the accumulation of the Atf1 protein (Figure 4) (Lawrence et al., 2009). Increased Atf1 levels lead to further recruitment of the TF at its cognate sequence, increased transcription of Atf1 targets and eventually oxidative stress resistance (Chen et al., 2003; Lawrence et al., 2007). 


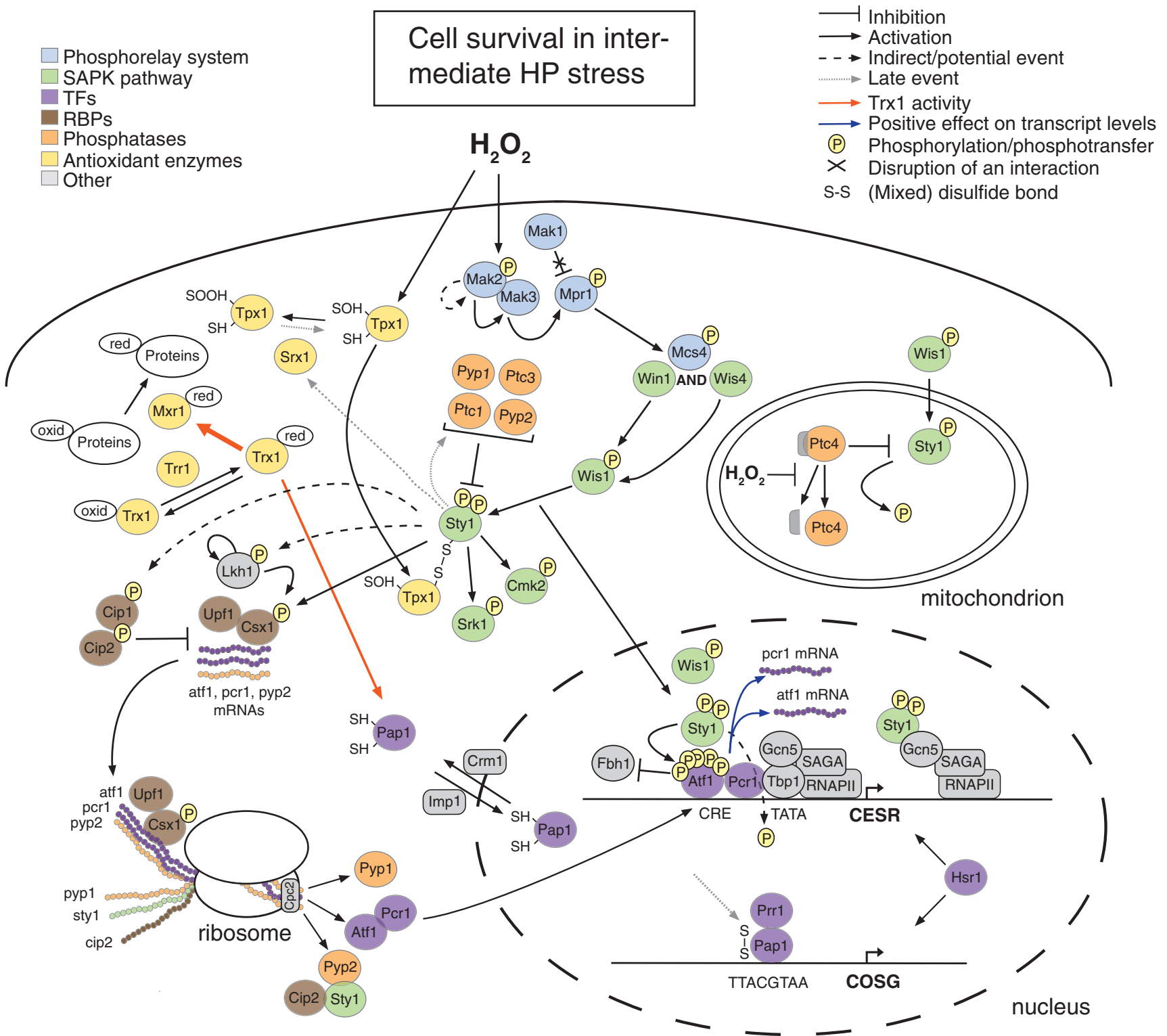

Figure 4. Overview of molecular events that promote cell survival upon intermediate oxidative stress $(0.25-1.0 \mathrm{mM}$ HP). The thickness of the arrows associated with the activity of thioredoxin (Trx1) provides a quantitative indication of the relative reaction rates. CESR, core environmental stress response genes; COSG, core oxidative stress genes; CRE, cAMP-responsive element; TATA, TATA/Goldberg-Hogness box (5'-TATAA-3'); RNAPII, RNA polymerase II; SAGA, Spt-Ada-Gen5-Acetyltransferase; oxid, oxidized; red, reduced. Additional graphical representations are explained in the upper left and right parts of the figure.

In the nucleus Atf1 can form a heterodimer with a second basic-leucine zipper (bZIP) TF, namely Pcr1/Mts2 (Watanabe \& Yamamoto, 1996). Atf1 and Pcr1 have overlapping binding sites (i.e. CRE site: 5'-ATGACGT-3') and exist as phosphoproteins at the promoters of core stress (i.e. CESR) genes even in unstressed conditions (Figures 4 and 6) (Eshaghi et al., 2010; Kanoh et al., 1996; Lawrence et al., 2007; Reiter et al., 2008; Sansó et al., 2008; Wahls \& Smith, 1994). Specifically upon oxidative stress, hyper-phosphorylation of Atf1 is accompanied by the Sty1-dependent dephosphorylation of Pcr1 (Figure 4) (Sansó et al., 2008).

Atf1 and Pcr1 regulate gene transcription either as a heterodimer or homodimer (Eshaghi et al., 2010). In conditions of HP stress the Atf1/Pcr1 heterodimer is primarily involved in the induction of Sty1-dependent genes, while its role in gene repression is minor (Eshaghi et al., 2010; Sansó et al., 2008). Although the heterodimer binds to the CRE site with much higher affinity than either homodimer complex (Kon et al., 1997), there are genes that are solely dependent on Atf1 and, to a lesser extent, on Pcr1 (Eshaghi et al., 2010; Reiter et al., 2008; Sansó et al., 2008). In particular, in response to intermediate stress $(0.5 \mathrm{mM} \mathrm{HP})$ the transcriptional regulation of 110,38 and 10 genes is, respectively, driven by Atf1/Pcr1, Atf1 and Pcr1 (Eshaghi et al., 2010). The requirement of an intact Atf1/Pcr1 heterodimer for Atf1 stabilization has been a topic of debate. The Sty1-mediated phosphorylation of Atf1 and heterodimerization of the latter with Pcr1 were initially suggested to increase the stability of the Atf1 protein (Lawrence et al., 2007). However, in a later study the role of transcription and/or translation was proposed to be of higher importance for the regulation of Atf1 levels (Sansó et al., 2008).

Pap1 encodes an AP-1-like TF, which contains a bZIP DNA-binding domain (Turner \& Tjian, 1989) and shares 


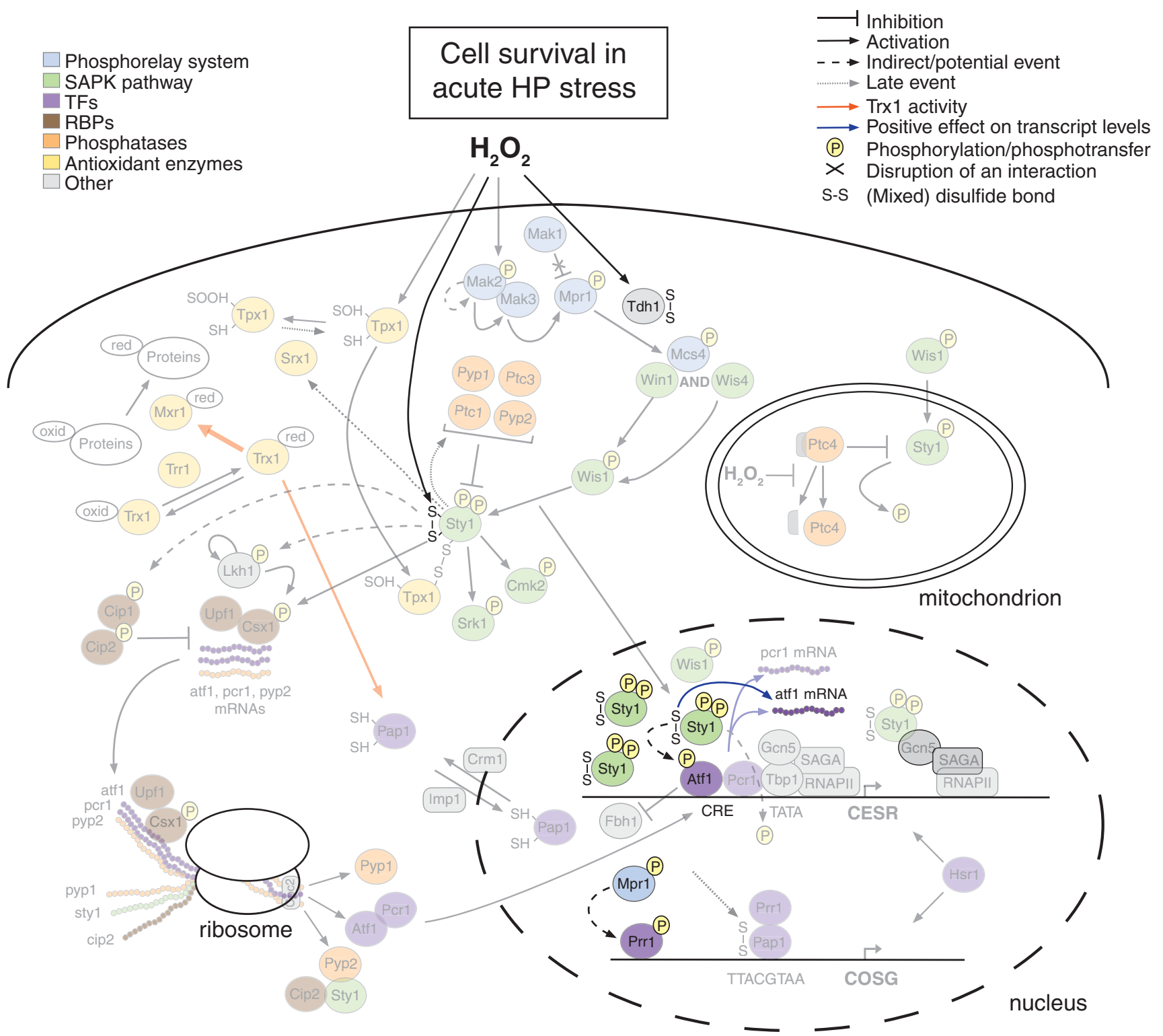

Figure 5. Molecular mechanisms that are specifically employed upon exposure to acute oxidative stress conditions (>1.0 mM HP). To emphasize molecular events that occur specifically in extreme - but not intermediate - HP stress, many branches of the demonstrated molecular network appear faded. Most of these branches remain, however, active even in very high HP doses (e.g. the SAPK pathway and the multistep phosphorelay sytem) (Quinn et al., 2002, 2011). Whether other faded parts of the figure represent active events in very high HP doses awaits validation (e.g. the (de)stabilization effects of the Csx1, Upf1, Cip1 and Cip2 RNA-binding proteins). The different graphical representations of the figure are explained in the upper left and right parts.

structural and DNA-binding properties with the mammalian c-Jun protein and the YAP1 TF in budding yeast (Table 1) (Moye-Rowley et al., 1989; Kouzarides \& Ziff, 1988). Atf1 and Pap1 regulate the expression of distinct and overlapping sets of genes in response to increasing HP levels (Bozonet et al., 2005; Chen et al., 2008). However, as described above, the role of the Atf1 is more profound in the response to intermediate HP concentrations, while Pap1 activates target genes primarily in response to low HP levels (Quinn et al., 2002).

Previous HP stress studies have suggested a role for Sty 1 in the regulation of Pap1 (Quinn et al., 2002). Although Pap1 appears to be phosphorylated after treatment of fission yeast cells with the microtubule depolymerizing agent thiabendazole (Wilson-Grady et al., 2008), upon intermediate and acute HP stress Sty1 has been hypothesized to exert its effects on
Pap1 through indirect mechanisms, with the possibility of direct phosphorylation of Pap1 by Sty1 remaining elusive (Quinn et al., 2002; Toone et al., 1998; Vivancos et al., 2004). Specifically, Atf1 could negatively regulate Pap 1 target genes by direct binding to their promoters, or alternatively, the phosphorylation state of Atf1 may affect Pap1 localization and/or activity (Quinn et al., 2002).

Moreover, Sty1-dependent regulation of Pap1 could be alternatively exerted through the activity of the Srx1 sulfiredoxin. Upon intermediate stress Tpx1 is temporarily inactivated through hyper-oxidation (Bozonet et al., 2005; Vivancos et al., 2005). Sty1-mediated induction of srxl expression results in the reactivation of the Tpx1 peroxidase activity (i.e. Srx1-mediated catalytic conversion of Cys-48-SOOH to Cys-48-SOH in the Tpx1 protein) and, thus, the activation and nuclear accumulation of Pap1 


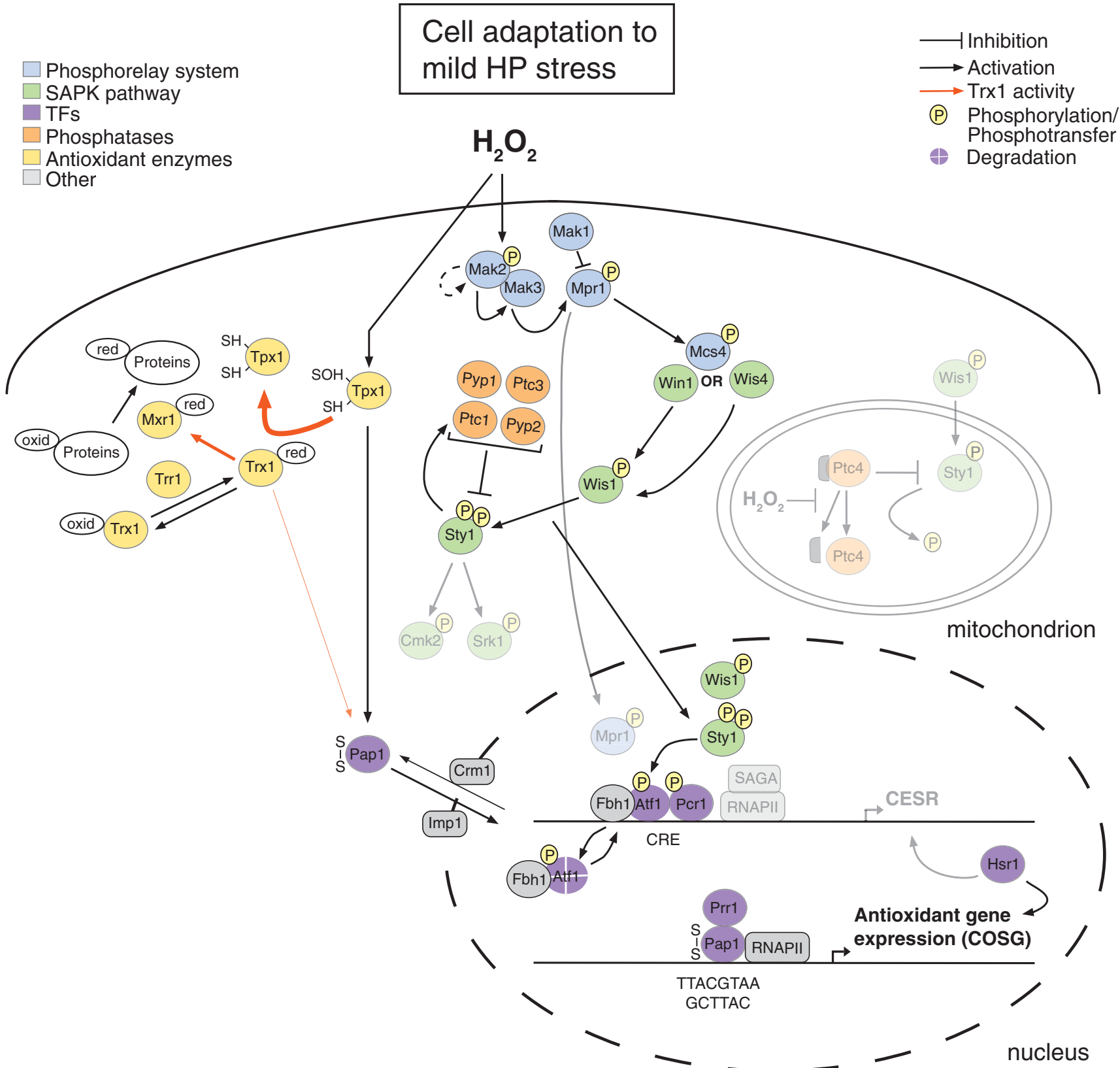

Figure 6. Overview of molecular events that enable cellular adaptation to mild oxidative stress $(<0.25 \mathrm{mM}$ HP). Faded parts of the figure correspond to events, whose occurrence remains elusive in low HP doses (e.g. the Ptc4-mediated regulation of Sty1 and the Sty1-mediated phosphorylation of the Srk1 and Cmk2 MAPKAPKs). The thickness of the arrows associated with the activity of thioredoxin (Trx1) provides a quantitative indication of the relative reaction rates. In the presence of low HP levels, the import of the Pap1 TF into the nucleus predominates the export to the cytoplasm, a fact indicated by a thicker arrow for Pap1 import. CESR, core environmental stress response genes; COSG, core oxidative stress genes; CRE, cAMPresponsive element; RNAPII, RNA polymerase II; SAGA, Spt-Ada-Gcn5-Acetyltransferase; oxid, oxidized; red, reduced. Additional graphical representations are explained in the upper left and right parts of the figure.

Table 1. Conservation of major stress transcription factors in yeasts and humans. The YOGY resource (Penkett et al., 2006) with default query settings was used to retrieve the budding yeast and human orthologs for the three transcription factors that are involved in the fission yeast response to oxidative stress. Name descriptions for budding yeast and human orthologs were extracted from the SGD (Cherry et al., 2012) and Ensembl databases (Flicek et al., 2012) respectively.

\begin{tabular}{lllll}
\hline S. pombe & S. cerevisiae & \multicolumn{1}{c}{ Name Description } & H. sapiens & \multicolumn{1}{c}{ Name Description } \\
\hline Atf1 and Pcr1 & ACA1 & ATF/CREB Activator & ATF2, 3, 7 & Activating TF 2, 3, 7 \\
& CST6 & Chromosome STability & BATF, BATF3 & bZIP ATF-like, ATF-like 3 \\
& & & JDP2 & Jun Dimerization Protein 2 \\
& & & FOS & FBJ murine osteosarcoma viral oncogene homolog \\
& & & FOSL1, 2 & XOS-Like antigens 1, 2 \\
Pap1 & YAP1 & Yeast AP-1 & NBP1 & NA Binding Protein 1 \\
Prr1 & SKN7 & Suppressor of Kre Null & & \\
& SFL1 & Suppressor gene for Flocculation & & \\
& HSF1 & Heat shock TF & & \\
\hline
\end{tabular}


(Bozonet et al., 2005; Vivancos et al., 2005). These events occur presumably late in the response to intermediate HP stress and constitute a positive feedback mechanism for the regulation of the Pap1 activity through the Sty1-Atf1 pathway (Figure 4) (Chen et al., 2008).

\section{Sty1 exerts additional nuclear roles}

Interestingly, Sty 1 is recruited at both the promoter and coding regions of Atf1/Pcr1-dependent genes (Figure 4) (Eshaghi et al., 2010; Reiter et al., 2008). Sty1 recruitment to the Atf1/Pcr1-bound HP-induced genes shows an apparent dependence on the heterodimer, but this is not the case for the SAPK recruitment at the bound-unresponsive genes (Eshaghi et al., 2010). It has, therefore, been speculated that upon HP stress other factors are bound at the promoters of the Atf1/ Pcr1-bound HP-unresponsive genes, and these factors suppress the activity of Atf1/Pcr1 and recruit Sty1 to the promoters of stress genes when Atf1 or Pcr1 is absent (Eshaghi et al., 2010).

Sty1 recruitment at stress promoters suggests an active role of the SAPK in transcription regulation (Reiter et al., 2008). Indeed, the phosphorylated form of the Sty1 (and Atf1) protein enables transcription initiation from stress promoters by triggering the recruitment of the RNA polymerase II (RNAPII), the TATA binding protein (Tbp1) and the Spt-AdaGcn5-Acetyltransferase (SAGA) complex (Figure 4) (Sansó et al., 2011b). To allow transcription progression, Atf1 or RNAPII recruit the Gcn5/Kat2 histone acetyltransferase, which facilitates RNAPII to escape stress promoters by acetylating the nucleosomal histones located downstream of the transcription start site. Notably, RNAPII drags Gen5 into the coding region of CESR genes during transcription elongation (Figure 4) (Sansó et al., 2011b).

Nuclear Sty1 employs additional mechanisms to increase the levels of the Atf1 protein, including the prevention of destabilization of the atfl mRNA. In response to very high HP levels $(>1.0 \mathrm{mM}$ ), two redox-sensitive cysteine residues (Cys153 and Cys-158) of the cytoplasmic Sty1 protein form an intramolecular disulfide bond on its surface (Figure 5) (Day \& Veal, 2010). The reversible oxidation of these cysteine residues prevents the HP-induced destabilization of the atf 1 transcript in the nucleus (Day \& Veal, 2010). In addition, transcription of atfl and pcrl is under the control of Sty1 (Chen et al., 2003), and the Atf1/Pcr1 heterodimer binds at the promoters of both genes, thus, forming a positive feedback loop (Figure 4) (Eshaghi et al., 2010). There also exists evidence for the Sty1-mediated stabilization of the atfl and pcrl transcripts at the post-transcriptional level (see the next section).

\section{Sty1 regulates gene expression at the post-transcriptional level}

In response to elevated HP levels Sty1 not only coordinates stress-mediated changes at the transcriptional level (Chen et al., 2008), but it also regulates gene expression at the posttranscriptional level through the direct or indirect control of RBP and PK activities (Park et al., 2003; Rodríguez-Gabriel \& Russell, 2008). Specifically upon intermediate oxidative stress, the Csx1 RBP directly binds and stabilizes mRNAs of several genes that are under the transcriptional control of Atf1 (e.g. pyp 2, atf1 and pcrl) (Figure 4) (Rodríguez-Gabriel et al., 2003). Interestingly, the mRNA levels of papl and prrl are not affected in $\operatorname{csxl}$ deletion mutants, and Csx 1 appears to have additional functions that do not involve Atf1 (RodríguezGabriel et al., 2003).

The Sty1 SAPK directly or indirectly controls the phosphorylation status of the Csx 1 protein, which can accept phosphate groups at four specific serine residues (Ser-42, -54, -291 and -455) (Rodríguez-Gabriel et al., 2003). Phosphorylation of Csx1 is, however, not critical for its function and other protein modifications could play a role in defining the specificity of its activity (Rodríguez-Gabriel et al., 2003). Csx 1 and Sty1 have independent functions in stress resistance, but both increase the specificity of the oxidative stress response by positively controlling the stability of the atfl transcript (Figure 4). The role of Sty1 in atfl mRNA stabilization is though more profound than that of Csx1 (Day \& Veal, 2010; Rodríguez-Gabriel et al., 2003).

Upon intermediate oxidative stress two other RBPs, Cip1 and Cip2, counteract the function of Csx1 to control the stability of cellular mRNAs. The three RBPs do not form a stable complex, but interact through their association with a transcript. The Cip1 and Cip2 proteins are cytoplasmic and become heavily phosphorylated upon HP stress in a Sty1dependent manner (Figure 4) (Martín et al., 2006). Although the mRNA levels of cip1, but not those of cip2, increase in the presence of elevated HP levels (Chen et al., 2003), Cip2, and to a lesser degree Cip1, require Atf1 for their function (Martín et al., 2006). Since constitutively high levels of the Atf1 protein are not beneficial for cellular physiology (Day \& Veal, 2010), the Sty1-dependent activation of two RBPs that counteract the stabilization effects of Csx1 suggests a mechanism to tightly control the mRNA levels of atfl.

Unexpectedly, upon oxidative stress the conserved RNA helicase Upf1, which is essential for the fission yeast nonsense-mediated mRNA decay (NMD) pathway, cooperates with Csx 1 to stabilize the atfl and pcrl mRNAs (RodríguezGabriel et al., 2006). The two RBPs exert their stabilization effects through the same pathway, but unlike Csx1, Upf1 does not interact directly with the atfl transcript (Figure 4). Instead Upf1 positively regulates mRNA stability by counteracting the activities of Cip1 and Cip2. Alternatively, Upf1 could mediate the degradation of a yet unknown transcript that harbors premature termination codon(s) and encodes a negative effector of the Csx1-Upf1 pathway (RodríguezGabriel et al., 2006). Another NMD factor, namely Upf2, seems to also play a role in the stress-mediated control of mRNA stability (Rodríguez-Gabriel et al., 2006).

It has been speculated that HP stress activates destabilizing factors or mRNA degradation pathways that are supported by Cip1 and Cip2 and counteracted by Csx1 and Upf1. An alternative hypothesis supports the idea that HP triggers direct transcript destabilization, which is prevented by Csx 1 and Upf1 binding on cellular mRNAs (Rodríguez-Gabriel et al., 2003, 2006). In either scenario, Cip1 and Cip2 counteract the stabilization activities of Csx 1 and Upf1. According to another interesting scenario, Cip1 and Cip2 could regulate the trafficking of transcripts in the cytoplasm and presumably contribute to the assembly of stress granules (SGs) 
(Martín et al., 2006). SGs are non-membranous structures, composed of messenger ribonuleoprotein particles (mRNPs) that form in the cytoplasm in response to diverse stress types (Anderson \& Kedersha, 2009).

All eukaryotes possess LAMMER kinases (LKs), which are proteins with the characteristic EHLAMMERILG motif in a kinase subdomain (Lee et al., 1996; Yun et al., 1994). LKs show dual substrate specificity and are capable of autophosphorylation (Howell et al., 1991). In addition to its diverse physiological roles, the single fission yeast LK, namely Lkh1/Kic1 (Kim et al., 2001), phosphorylates Csx1 and, thus, plays an essential role in the stress-induced posttranscriptional regulation of gene expression (Kang et al., 2007). Lkh1-mediated phosphorylation of Csx 1 results in increased stability of the atfl transcript and induction of diverse stress-defense genes, including sodl and cttl/ctal (James et al., 2009; Park et al., 2003). The stress-related activity of Lkh1 is presumably regulated through the Sty1 pathway (Figure 4) (Kang et al., 2007).

Co-translational assembly of protein complexes is a common albeit specific phenomenon in fission yeast (Duncan \& Mata, 2011). Sty1 has been shown to interact with three mRNAs, cip2 and pyp2 and its own mRNA (Figure 4) (Duncan \& Mata, 2011). The association of Sty1 with two of its targets (i.e. Cip1 and Pyp2) (Figure 4) (Wilkinson et al., 1996; Martín et al., 2006) presumably reflects the necessity to tightly regulate the stress-related functions of the Cip2 RBP and the Pyp2 protein phosphatase (Duncan \& Mata, 2011). Similarly, the concomitant assembly of the Atf1/Pcr1 heterodimer (Figure 4) (Duncan \& Mata, 2011) supports the idea of a cross-protective relationship between Atf1 and Pcr1, whereby the two TFs protect each other from ubiquitination and degradation (Lawrence et al., 2007).

\section{The Sty 1 regulatory signals on translation}

In response to oxidative stress global translation is halted through phosphorylation of the translation initiation factor $2 \alpha$ (eIF2 $\alpha$ ) (Dever et al., 2007). As shown in S. cerevisiae this post-translational event occurs at the onset of the stress response in a Gcn2/Ppk28-dependent manner (Mascarenhas et al., 2008). Another eIF2 $\alpha$ kinase, Hri2, becomes also critical for survival upon long exposure of fission yeast cells to stress (Dunand-Sauthier et al., 2005; Zhan et al., 2002). Stress-induced activation of the Sty1 pathway triggers translation re-initiation by negatively regulating the closely related pathways of the Gcn2 and Hri2 kinases (Berlanga et al., 2010; Dunand-Sauthier et al., 2005). However, the corresponding molecular mechanisms remain unclear. It has been speculated that downstream components of the fission yeast SAPK pathway regulate the kinase activities of Hri2 and Gcn2 through direct interaction. Alternatively, the Sty1mediated ROS detoxification has been suggested as a means to indirectly inhibit the two kinases (DunandSauthier et al., 2005).

Many eukaryotic species encode PKs that are activated through phosphorylation by an upstream MAPK, i.e. MAPKactivated protein kinases (MAPKAPKs) (Bilsland et al., 2004). These proteins have been linked to the post-transcriptional control of gene expression in mammals and yeast (Dahlkvist et al., 1994; Neininger et al., 2002). Fission yeast encodes two MAPKAPKs (Srk1/Mkp1 and Cmk2/Mkp2), which are calmodulin-dependent, dual specificity protein kinases (Alemany et al., 2002). Upon oxidative stress both MAPKAPKs are activated by phosphorylation in a Sty1-dependent manner (Figure 4), with the Srk1-Sty1 interaction being stronger than that of Cmk2-Sty1 (Asp \& Sunnerhagen, 2003). There is no apparent link between the post-stress recovery of general translation and the kinase activity of Srk1 or Cmk2 (Asp et al., 2008). However, based on the fact that RCK2, the Cmk2 ortholog in budding yeast, phosphorylates in vitro the eukaryotic translation elongation factor 2 (eEF2), Cmk2 has been suggested as a mediator of the Sty1 regulatory signals on translation (Sanchez-Piris et al., 2002).

Additionally, Sty 1 has been shown to directly interact with some translation factors, including eEF2 and the eukaryotic initiation factor 3a (eIF3a) (Asp et al., 2008). When HP levels are higher than normal, the Sty1-eIF3a interaction weakens, but the Sty1-eEF2 association remains stable (Asp et al., 2008). Global translation can be maintained without eIF3a (Asp et al., 2008) and the composition of eIF3 varies in different stress conditions to serve global and specific translation demands (Zhou et al., 2005). These findings support the idea that upon oxidative stress Sty1 allows specific cellular needs to be met by affecting primarily the initiation rather than the elongation phase of translation (Dunand-Sauthier et al., 2005).

Another mechanism employed by Sty 1 to alter translation initiation and affect protein expression in response to oxidative stimuli involves the inhibition of the proteolytic cleavage of the Pabp polyA-binding protein, which is involved in shortening the polyA tail of cellular transcripts (Weeks et al., 2006).

\section{Cell cycle progression in oxidative stress: the role of Sty1}

Treatment of $S$. pombe cells with intermediate HP doses $(0.5 \mathrm{mM})$ causes an arrest at the $\mathrm{G} 2$ phase of the cell cycle (Chen et al., 2008). Upon stress a common cellular mechanism to slow down the mitotic cell cycle progression at the G2/M transition involves the Sty1-mediated regulation of the Srk1 MAPKAPK (Asp \& Sunnerhagen, 2003). Srk1 regulates the onset of mitosis and exhibits an inhibitory effect on the Cdc2-activating phosphatase Cdc25 (Lopez-Aviles et al., 2005). Indeed, the transcript levels of srkl, but not those of the second fission yeast MAPKAPK (i.e. cmk2), become highly increased after environmental perturbations, including oxidative stress (Chen et al., 2003; Smith et al., 2002). Notably, the expression of $s r k l$ is regulated by the mitochondrial TF Mtf1, which has been previously linked to cell cycle control (Sun et al., 2011).

Moreover, it has been shown that the overexpression of a constitutively active form of the Cmk2 MAPKAPK results in low Cdc2 activity and eventually in G2 arrest (Alemany et al., 2002; Rasmussen \& Rasmussen, 1994). In response to oxidative stress, Sty1 binds and phosphorylates Cmk2 at the single phosphorylation site (Thr-411) of the protein 
(Sanchez-Piris et al., 2002). Cmk2 levels change during the cell cycle, with $\mathrm{Cmk} 2$ expression peaking at the end of mitosis and becoming negligible at the entry into the G2 phase (Alemany et al., 2002). Due to its Sty1-dependent regulation and its localization at the growth sites of the cell, Cmk2 has been suggested to play a role in cell morphogenesis when intracellular ROS levels are high (Alemany et al., 2002).

\section{Control of Sty1 signaling during stress recovery}

If the cells are able to remediate their environment, or the stress is attenuated in one way or another, deactivation of repair pathways and restoration of growth processes must also be achieved. Therefore, the amplitude and duration of the MAPK-mediated signaling are two parameters that become tightly regulated as part of the oxidative stress response (Kyriakis \& Avruch, 2001). Towards this direction, specific protein phosphatases dephosphorylate and deactivate the Sty1 SAPK to initiate the recovery phase of the response. Four PP2C-type serine/threonine phosphatases (Ptc1-4) and two tyrosine phosphatases (Pyp1-2) act in a condition-specific manner to remove phosphates from the Thr-171 and Tyr-173 residues of Sty1, respectively (Millar et al., 1995; Nguyen \& Shiozaki, 1999; Shiozaki \& Russell, 1995b). For instance, Pyp1 and Ptc2 are constitutively expressed and deactivate Sty1 during normal growth (Gaits et al., 1997; Shiozaki \& Russell, 1995b). In heat stress the binding between Sty1 and Pyp1 is abolished to activate the MAPK, which is subsequently deactivated by Ptc1 and/or Ptc3 (Nguyen \& Shiozaki, 1999).

Upon oxidative stress Pyp1, Pyp2, Ptc1 and Ptc3 deactivate Sty1 after the stress-related transcriptional program has been launched (Chen et al., 2003; Gaits et al., 1997; Millar et al., 1995). Interestingly, the attenuation of Sty1 signaling by Pyp1, Pyp2 and Ptc1 is mediated through the Sty1-Atf1 pathway, thus forming a negative feedback loop (Figure 4) (Degols et al., 1996; Shiozaki \& Russell, 1996; Wilkinson et al., 1996). Specifically upon HP stress the Ptc4 phosphatase deactivates the mitochondrial pool of activated Sty1 in a mechanism that involves the inhibition of HP-mediated cleavage of the Ptc4 mitochondrial targeting sequence (Figure 4) (Di et al., 2012). In oxidative stress conditions the full-length form of the Ptc4 protein and the mitochondrial fraction of Sty1 are strongly associated with mitochondrial membranes (Di et al., 2012). Although the scenario that Sty1 shuttles through mitochondria in addition to its nuclear translocation awaits verification, the role of Sty1 in the mitochondrion should be related to cellular respiration (Di et al., 2012).

Furthermore, Sty1-mediated signaling is regulated at the translational level through the activity of the RACK1 ortholog $\mathrm{Cpc} 2 / \mathrm{Rkp} 1$, which is a component of the 40S ribosomal subunit and has an important role in cell cycle progression (Nunez et al., 2010). In response to diverse stress conditions, including elevated HP levels, Cpc2 favors the translation of some downstream Sty1 targets, such as the mRNAs of pypl, pyp2, atfl and cttl (Figure 4) (Núñez et al., 2009). Although the function of Atf1 as a transcriptional activator in response to stress is not compromised in $c p c 2$ deletion mutants (Núñez et al., 2009), Cpc2 assists the cell to scavenge elevated ROS levels through efficient translation of the $c t t 1$ mRNA. Given that the Pmk1 and Sty1 MAPK pathways share regulatory links (i.e. the Pyp1, Pyp2 and Ptc1 phosphatases), the translational control of tyrosine phosphatases by $\mathrm{Cpc} 2$ should allow for the amplitude and duration of signaling mediated by these two pathways to be tightly controlled and coordinated.

\section{Cellular adaptation to mild oxidative stress}

In the presence of non-physiological but low HP concentrations, there is an increased cellular demand in enzymes that scavenge ROS and promote redox homeostasis (e.g. Ctt1, Trx 1 and Trr1/Caf4). The Pap1 TF is thus activated to regulate the expression of COSG, which mainly encode enzymes that confer antioxidant protection (Chen et al., 2008).

Pap1 has a nuclear import signal (NLS) and a nuclear export signal (NES), which are recognized by the importin- $\alpha$ Imp1 (Umeda et al., 2005) and the exportin Crm1/Caf2 (Kudo et al., 1999) respectively. Under normal conditions, Pap1 is primarily cytosolic because Crm1 binds the NES of the Pap1 protein and mediates its nuclear export (Castillo et al., 2002; Vivancos et al., 2004).

Upon exposure to mild HP concentrations $(0.07-0.25 \mathrm{mM})$ Tpx1 mediates the activation of Pap1 in a process that requires both the peroxidatic and resolving cysteines of the enzyme (Vivancos et al., 2005). The process presumably involves the formation of an intermolecular disulfide bond between Tpx1 (Cys-48) and Pap1 (Cys-501 or Cys-532) (Vivancos et al., 2005). Transduction of the HP signal from Tpx1 to Pap1 induces a conformational change to the TF, which results in the dissociation of Pap1 from the Crm1-Hbal nuclear export machinery (Figure 6) (Castillo et al., 2002, 2003; Vivancos et al., 2004). Notably, Tpx1 and Pap1 undergo thiol oxidation at similar times with other protein thiols (García-Santamarina et al., 2011).

After its nuclear accumulation, Pap1 regulates the expression of most COSG (Figure 6) (Chen et al., 2008) through binding on the consensus nucleotide sequence $5^{\prime}$ TTACGTAA-3' (Fujii et al., 2000), which slightly deviates from the consensus DNA sequence for bZIP motifs (Fujii et al., 1998). Moreover, the promoter regions of genes being under the control of Pap1 in mild HP (and menadione) stress are enriched for a distinct sequence motif (5'-GCTTAC-3') (Chen et al., 2008). To some extent, induction of Pap1 targets requires the formation of a heterodimer between the oxidized Pap1 protein and Prr1 (Figure 6) (Calvo et al., 2012; Chen et al., 2008). It has been speculated that oxidation of Pap1 exposes a Prr1-interacting domain, which leads to the enhancement of the Pap1 affinity for antioxidant promoters (Calvo et al., 2012).

In fission yeast, Int6 is a conserved subunit of the eIF3 and has been linked to the promotion of general translation and proper Sty1-mediated signaling in response to different types of stress (Udagawa et al., 2008). A truncated version of the Int6 subunit, Int6CT, has been shown to activate Pap1dependent transcription by an alternative mechanism, which does not involve a redox-mediated conformational change or nuclear accumulation of the Pap1 protein (Jenkins et al., 2005). However, the mechanism itself and whether the 
Int6CT-mediated activation of Pap1 reflects an aspect of normal cell physiology remain elusive.

In mild HP stress conditions the multiple phosphorelay system and the SAPK pathway are active, and either of the Wis1 and Win1 MAPKKKs interacts with Mcs4 to transduce the stress signal to Sty1 - as opposed to intermediate HP stress, where both MAPKKKs are required for signal transduction (Quinn et al., 2002). Moreover, it has been shown that the levels of active Sty1 increase with increasing HP concentrations (Quinn et al., 2002). Upon mild HP stress the levels of active Sty1 are thus expected to be low. The phosphorylation status of Atf1 should, therefore, be kept close to the basal levels (Lawrence et al., 2007), with the protein being under continual turnover through the ubiquitinproteasome system (Figure 6) (Lawrence et al., 2009). Although in the presence of low HP concentrations the levels of active Sty 1 are low and the protein is not recruited to stress promoters, Atf1 should be able to support the weak expression of CESR genes, as previously reported for unstressed cells (Reiter et al., 2008).

\section{Different fission yeast responses to oxidative stress of different intensity: a summarized mechanistic view}

As presented throughout the manuscript, fission yeast employs different regulatory pathways and TFs to mount an effective response to oxidative stress of different intensity. This is achieved by two major mechanisms. Firstly, the Mak2Mak3-Mpr1-Mcs4 multistep phosphorelay system specifically senses oxidative stress signals and relays them to the Sty1 MAPK (Buck et al., 2001; Quinn et al., 2011). Secondly, the redox coupling between the thioredoxin activity of the $\operatorname{Trx} 1$ protein and the thioredoxin peroxidase activity of the Tpx1 enzyme defines the balance between the activation of two independent pathways, the Pap1 and the Sty1 pathway.

Upon exposure to intermediate HP doses $(0.25-1.0 \mathrm{mM})$ Tpx1 is required for the activation of Sty1, which initiates a transcriptional program that promotes cell survival (Veal et al., 2004). In these conditions, the temporal inactivation of Tpx1 through hyper-oxidation (Bozonet et al., 2005; Vivancos et al., 2005) is of particular importance for the response, as it allows Trx 1 to reduce the Pap1 TF and other oxidized cellular proteins with a key role in damage repair (e.g. Mxr1/MsrA, peptide methionine sulfoxide reductase) (Figure 4) (Day et al., 2012). In this manner the Pap1 pathway is "switched off" (Bozonet et al., 2005; Vivancos et al., 2005) and the restricted capability of the HP-damaged translational machinery to de novo synthesize protective molecules is overcome (Grant, 2011). Although Pap1 (together with the Prr1 RR) is the main regulator of gene expression in mild HP stress $(<0.25 \mathrm{mM})$, the expression of a small set of genes (Figure 4) depends on the cooperative activity of these two TFs even in intermediate HP stress (Chen et al., 2008; Quinn et al., 2011) (Quinn et al., 2002).

Upon acute exposure ( $>1.0 \mathrm{mM} \mathrm{HP})$, the Prrl RR and its regulation through the phosphorelay system are presumably crucial for the required stress-induced gene regulation (Quinn et al., 2011). At very high HP concentrations, Sty1 phosphorylation is increased, but the Atf1-dependent gene transcription is only slightly induced (Chen et al., 2008). This could be explained by the overall inhibition of cellular transcription, which presumably results from the extremity of the stress condition. The sensitivity of the Sty1 protein to the HP-induced oxidation could also partly explain the reduction in Atf1-mediated transcription that is observed upon exposure to high HP levels (Day \& Veal, 2010).

Upon mild HP stress $(<0.25 \mathrm{mM})$, Tpx 1 sequesters Trx 1 and triggers the oxidation-mediated activation of the Pap1 TF (Bozonet et al., 2005; Chen et al., 2008; Vivancos et al., 2005). By sequestering Trx $1, \operatorname{Tpx} 1$ prevents the $\operatorname{Trx} 1-$ mediated reduction of Pap1 and, thus, reinforces the Pap1mediated gene regulation (Figure 6) (Day et al., 2012). At low HP levels Pap1 together with Prr1 regulate the expression of antioxidant genes (Figure 6), with the role of the former being more pronounced than that of the latter (Chen et al., 2008). Importantly, the Mcs4-mediated signaling to Sty1 and the Sty1-Atf1 pathway are essential at intermediate to extreme HP doses (0.25-6.0 mM) (Chen et al., 2008; Quinn et al., 2011) but possess only a marginal role in mild stress conditions (0.07-0.25 mM HP) (Figures 4, 5 and 6) (Chen et al., 2003, 2008; Quinn et al., 2011, 2002).

From the above it seems that the Prr1 RR is essential for the transcriptional response to a wide range of HP concentrations (Figures 4-6) (Chen et al., 2008; Quinn et al., 2011). In addition to its Pap1-supporting role (Chen et al., 2008; Calvo et al., 2012), Prr1 induces stress genes independently of Sty1 (Ohmiya et al., 1999, 2000) and it supports the induction of Atf1-dependent genes (Calvo et al., 2012; Greenall et al., 2002). No orthologous protein of Prr1 exists in humans (Penkett et al., 2006), while one of the Prr1 orthologs in budding yeast, SKN7, assists the YAP1 TF (i.e. the ortholog of Pap1 in S. cerevisiae) (Table 1) to induce some of its targets (Ikner \& Shiozaki, 2005).

Transcriptional regulation in HP stress becomes even more complex as new regulators are being discovered. For instance, the expression of the Hsr1 zinc-finger TF is under the control of all four regulators, Sty1-Atf1, Pap1 and Prr1, and it appears to support global gene expression upon oxidative stress (Chen et al., 2008). It has been proposed that Hsr1 is a part of a positive feedback for the response to HP stress, since it is required for the full induction of genes that are regulated by different TFs (i.e. COSG regulated by Pap1 and/or Prr1 and CESR genes regulated by Atf1) (Figures 4 and 6) (Chen et al., 2008).

\section{Conclusions and perspectives}

Cellular stress responses represent an important interaction between the cell and its environment with implications on its behavior and survival. Stress responses may serve as models for signaling pathways in general, as for instance to understand how natural genetic variability affects intracellular signal processing. To understand the mechanisms involved in signaling for any agent, signal transducers and their physical interactions within the highly interactive networks of the cell have to be precisely defined. Moreover, construction of mechanistic models describing cellular signaling necessitates knowledge on the number, identity and subcellular 
localization of the PKs and additional proteins that are activated in response to a given stimulus.

In humans, redox imbalance is associated with aging, cancer, Alzheimer's and Parkinson's disease among others. Therefore, studies investigating the cellular mechanisms employed in response to oxidative stress have markedly increased in recent years, especially using model organisms. Fission yeast is well suited for such studies given the high degree of complexity and sophistication of the pathways it employs in response to oxidative stimuli. Despite the wealth of existing knowledge, some aspects of the fission yeast response to HP stress require further investigation. For instance, existing in silico predictions on Csx 1 targets (Pancaldi \& Bähler, 2011) and predicted interactions between the Mak1-3 proteins and a number of TFs (Pancaldi et al., 2012) need to be validated. To refine the current view of transcriptional regulation in oxidative stress, the binding specificities of the less studied transcriptional regulators that are highly induced upon HP exposure (Chen et al., 2003, 2008) need to be defined as well.

Nevertheless, the fission yeast oxidative stress response has been studied in sufficient detail to allow the development of new experimental and computational methods, which can be verified based on the framework of available knowledge. Understanding the fission yeast inner workings will empower research into more complex systems that control stress response networks in human cells.

\section{Declaration of interest}

The authors report no conflict of interest. This work was supported by the EU FP7 PhenOxiGEn (ref. no.: 223539) project.

\section{References}

Aguirre J, Rios-Momberg M, Hewitt D, Hansberg W. (2005). Reactive oxygen species and development in microbial eukaryotes. Trends Microbiol 13:111-18.

Alemany V, Sanchez-Piris M, Bachs O, Aligue R. (2002). Cmk2, a novel serine/threonine kinase in fission yeast. FEBS Lett 524:79-86.

Alex LA, Korch C, Selitrennikoff CP, Simon MI. (1998). COS1, a twocomponent histidine kinase that is involved in hyphal development in the opportunistic pathogen Candida albicans. Proc Natl Acad Sci USA 95:7069-73.

Anderson P, Kedersha N. (2009). Stress granules. Curr Biol 19:R397-8.

Asp E, Sunnerhagen P. (2003). Mkp1 and Mkp2, two MAPKAP-kinase homologues in Schizosaccharomyces pombe, interact with the MAP kinase Sty1. Mol Genet Genomics 268:585-97.

Asp E, Nilsson D, Sunnerhagen P. (2008). Fission yeast mitogenactivated protein kinase Sty1 interacts with translation factors. Eukaryotic Cell 7:328-38.

Berlanga JJ, Rivero D, Martin R, et al. (2010). Role of mitogen-activated protein kinase Sty1 in regulation of eukaryotic initiation factor 2alpha kinases in response to environmental stress in Schizosaccharomyces pombe. Eukaryotic Cell 9:194-207.

Bilsland E, Molin C, Swaminathan S, et al. (2004). Rck1 and Rck2 MAPKAP kinases and the HOG pathway are required for oxidative stress resistance. Mol Microbiol 53:1743-56.

Bozonet SM, Findlay VJ, Day AM, et al. (2005). Oxidation of a eukaryotic 2-Cys peroxiredoxin is a molecular switch controlling the transcriptional response to increasing levels of hydrogen peroxide. J Biol Chem 280:23319-27.

Brauer MJ, Huttenhower C, Airoldi EM, et al. (2008). Coordination of growth rate, cell cycle, stress response, and metabolic activity in yeast. Mol Biol Cell 19:352-67.
Brewster JL, de Valoir T, Dwyer ND, et al. (1993). An osmosensing signal transduction pathway in yeast. Science 259:1760-3.

Buck V, Quinn J, Soto Pino T, et al. (2001). Peroxide sensors for the fission yeast stress-activated mitogen-activated protein kinase pathway. Mol Biol Cell 12:407-19.

Calera JA, Calderone R. (1999a). Flocculation of hyphae is associated with a deletion in the putative CaHK1 two-component histidine kinase gene from Candida albicans. Microbiology 145:1431-42.

Calera JA, Calderone RA. (1999b). Identification of a putative response regulator two-component phosphorelay gene ( CaSSK1) from Candida albicans. Yeast 15:1243-54.

Calera JA, Herman D, Calderone R. (2000). Identification of YPD1, a gene of Candida albicans which encodes a two-component phosphohistidine intermediate protein. Yeast 16:1053-9.

Calvo IA, Garcia P, Ayte J, Hidalgo E. (2012). The transcription factors Pap1 and Prr1 collaborate to activate antioxidant, but not drug tolerance, genes in response to $\mathrm{H}_{2} \mathrm{O}_{2}$. Nucleic Acids Research 40:4816-24.

Castillo EA, Ayte J, Chiva C, et al. (2002). Diethylmaleate activates the transcription factor Pap1 by covalent modification of critical cysteine residues. Mol Microbiol 45:243-54.

Castillo EA, Vivancos AP, Jones N, et al. (2003). Schizosaccharomyces pombe cells lacking the Ran-binding protein Hbal show a multidrug resistance phenotype due to constitutive nuclear accumulation of Pap1. J Biol Chem 278:40565-72.

Castrillo JI, Zeef LA, Hoyle DC, et al. (2007). Growth control of the eukaryote cell: a systems biology study in yeast. J Biol 6:4.

Causton HC, Ren B, Koh SS, et al. (2001). Remodeling of yeast genome expression in response to environmental changes. Mol Biol Cell 12:323-37.

Cheetham J, Smith DA, da Silva Dantas A, et al. (2007). A single MAPKKK regulates the Hog1 MAPK pathway in the pathogenic fungus Candida albicans. Mol Biol Cell 18:4603-14.

Chen D, Toone WM, Mata J, et al. (2003). Global transcriptional responses of fission yeast to environmental stress. Mol Biol Cell 14:214-29.

Chen D, Wilkinson CR, Watt S, et al. (2008). Multiple pathways differentially regulate global oxidative stress responses in fission yeast. Mol Biol Cell 19:308-17.

Cherry JM, Hong EL, Amundsen C, et al. (2012). Saccharomyces Genome Database: the genomics resource of budding yeast. Nucleic Acids Research 40:D700-5.

Dahlkvist A, Sunnerhagen P. (1994). Two novel deduced serine/ threonine protein kinases from Saccharomyces cerevisiae. Gene 139:27-33.

Day AM, Veal EA. (2010). Hydrogen peroxide-sensitive cysteines in the Sty1 MAPK regulate the transcriptional response to oxidative stress. J Biol Chem 285:7505-16.

Day AM, Brown JD, Taylor SR, et al. (2012). Inactivation of a peroxiredoxin by hydrogen peroxide is critical for thioredoxinmediated repair of oxidized proteins and cell survival. Mol Cell 45:398-408.

Degols G, Russell P. (1997). Discrete roles of the Spc1 kinase and the Atf1 transcription factor in the UV response of Schizosaccharomyces pombe. Mol Cell Biol 17:3356-63.

Degols G, Shiozaki K, Russell P. (1996). Activation and regulation of the Spc1 stress-activated protein kinase in Schizosaccharomyces pombe. Mol Cell Biol 16:2870-7.

Desaint S, Luriau S, Aude J-C, et al. (2004). Mammalian antioxidant defenses are not inducible by H2O2. J Biol Chem 279:31157-63.

Dever TE, Dar AC, Sicheri F. (2007). 12 The eIF2a Kinases. Cold Spring Harbor Monograph Archive 48:319-44.

Derijard B, Hibi M, Wu IH, et al. (1994). JNK1: a protein kinase stimulated by UV light and Ha-Ras that binds and phosphorylates the c-Jun activation domain. Cell 76:1025-37.

Di Y, Holmes EJ, Butt A, et al. (2012). $\mathrm{H}_{2} \mathrm{O}_{2}$ stress-specific regulation of S. pombe MAPK Sty1 by mitochondrial protein phosphatase Ptc4. EMBO J 31:563-75.

Dunand-Sauthier I, Walker CA, Narasimhan J, et al. (2005). Stressactivated protein kinase pathway functions to support protein synthesis and translational adaptation in response to environmental stress in fission yeast. Eukaryotic Cell 4:1785-93.

Duncan CDS, Mata J. (2011). Widespread cotranslational formation of protein complexes. PLoS Genetics 7:e1002398. 
English J, Pearson G, Wilsbacher J, et al. (1999). New insights into the control of MAP kinase pathways. Exp Cell Res 253:255-70.

Erbel PJA, Card PB, Karakuzu O, et al. (2003). Structural basis for PAS domain heterodimerization in the basic helix-loop-helix-PAS transcription factor hypoxia-inducible factor. Proc Natl Acad Sci USA 100:15504-9.

Eshaghi M, Lee JH, Zhu L, et al. (2010). Genomic binding profiling of the fission yeast stress-activated MAPK Sty1 and the bZIP transcriptional activator Atf1 in response to $\mathrm{H} 2 \mathrm{O} 2$. PloS one 5:e11620.

Estruch F. (2000). Stress-controlled transcription factors, stress-induced genes and stress tolerance in budding yeast. FEMS Microbiol Reviews 24:469-86.

Finkel T, Holbrook NJ. (2000). Oxidants, oxidative stress and the biology of ageing. Nature 408:239-47.

Flicek P, Ahmed I, Amode MR, et al. (2012). Ensembl 2013. Nucleic Acids Res 41:D48-55.

Fujii Y, Ohira T, Kyougoku Y, et al. (1998). Crystallographic characterization of Pap1-DNA complex. Acta Cryst D54, 1014-16. [doi:10.1107/S0907444998003266].

Fujii Y, Shimizu T, Toda T, et al. (2000). Structural basis for the diversity of DNA recognition by bZIP transcription factors. Nat Struct Mol Biol 7:889-93.

Furukawa K, Hoshi Y, Maeda T, et al. (2005). Aspergillus nidulans HOG pathway is activated only by two-component signalling pathway in response to osmotic stress. Mol Microbiol 56:1246-61.

Gaits F, Degols G, Shiozaki K, Russell P. (1998). Phosphorylation and association with the transcription factor Atf1 regulate localization of Spc1/Sty1 stress-activated kinase in fission yeast. Genes Dev $12: 1464-73$

Gaits F, Shiozaki K, Russell P. (1997). Protein phosphatase 2C acts independently of stress-activated kinase cascade to regulate the stress response in fission yeast. J Biol Chem 272:17873-9.

Garcia-Santamarina S, Boronat S, Espadas G, et al. (2011). The oxidized thiol proteome in fission yeast. J Proteomics 74:2476-86.

Gasch AP, Spellman PT, Kao CM, et al. (2000). Genomic expression programs in the response of yeast cells to environmental changes. Mol Biol Cell 11:4241-57.

Grant CM. (2011). Regulation of Translation by Hydrogen Peroxide. Antioxid Redox Signaling 15:191-203.

Grant CM, Quinn KA, Dawes IW. (1999). Differential protein Sthiolation of glyceraldehyde-3-phosphate dehydrogenase isoenzymes influences sensitivity to oxidative stress. Mol Cell Biol 19:2650-6.

Greenall A, Hadcroft AP, Malakasi P, et al. (2002). Role of fission yeast Tup1-like repressors and Prr1 transcription factor in response to salt stress. Mol Biol Cell 13:2977-89.

Hohmann S. (2002). Osmotic stress signaling and osmoadaptation in yeasts. Microbiol Mol Biol Rev 66:300-72.

Howell BW, Afar DE, Lew J, et al. (1991). STY, a tyrosinephosphorylating enzyme with sequence homology to serine/threonine kinases. Mol Cell Biol 11:568-72.

Hughes TR, Marton MJ, Jones AR, et al. (2000). Functional discovery via a compendium of expression profiles. Cell 102:109-26.

Ikner A, Shiozaki K. (2005). Yeast signaling pathways in the oxidative stress response. Mutat Res-Fund Mol M 569:13-27.

Imamura A, Hanaki N, Umeda H, et al. (1998). Response regulators implicated in His-to-Asp phosphotransfer signaling in Arabidopsis. Proc Natl Acad Sci USA 95:2691-6.

James BP, James BP, Staatz WD, et al. (2009). Superoxide dismutase is regulated by LAMMER kinase in Drosophila and human cells. Free Radic Biol Med 46:821-7.

Jenkins CCL, Mata J, Crane RF, et al. (2005). Activation of AP-1dependent transcription by a truncated translation initiation factor. Eukaryotic Cell 4:1840-50.

Kang W-H, Park Y-D, Hwang J-S, Park H-M. (2007). RNA-binding protein Csx1 is phosphorylated by LAMMER kinase, Lkh1, in response to oxidative stress in Schizosaccharomyces pombe. FEBS Lett 581:3473-8.

Kanoh J, Watanabe Y, Ohsugi M, et al. (1996). Schizosaccharomyces pombe gad7+ encodes a phosphoprotein with a bZIP domain, which is required for proper G1 arrest and gene expression under nitrogen starvation. Genes to cells 1:391-408.

Kawasaki L, Sanchez O, Shiozaki K, Aguirre J. (2002). SakA MAP kinase is involved in stress signal transduction, sexual development and spore viability in Aspergillus nidulans. Mol Microbiol 45:1153-63.
Kim K-H, Cho Y-M, Kang W-H, et al. (2001). Negative Regulation of Filamentous Growth and Flocculation by Lkh1, a Fission Yeast LAMMER Kinase Homolog. Biochem Biophys Res Commun 289:1237-42.

Kon N, Krawchuk MD, Warren BG, et al. (1997). Transcription factor Mts1/Mts2 (Atf1/Pcr1, Gad7/Pcr1) activates the M26 meiotic recombination hotspot in Schizosaccharomyces pombe. Proc Natl Acad Sci USA 94:13765-70.

Kouzarides T, Ziff E. (1988). The role of the leucine zipper in the fos-jun interaction. Nature 336:646-51.

Kudo N, Taoka H, Toda T, et al. (1999). A novel nuclear export signal sensitive to oxidative stress in the fission yeast transcription factor Pap1. J Biol Chem 274:15151-8.

Kultz D. (2005). Molecular and evolutionary basis of the cellular stress response. Annu Rev Physiol 67:225-57.

Kyriakis JM, Avruch J. (2001). Mammalian mitogen-activated protein kinase signal transduction pathways activated by stress and inflammation. Physiol Rev 81:807-69.

Lackner DH, Schmidt MW, Wu S, et al. (2012). Regulation of transcriptome, translation, and proteome in response to environmental stress in fission yeast. Genome Biol 13:R25.

Lawrence CL, Jones N, Wilkinson CRM. (2009). Stress-induced phosphorylation of $\mathrm{S}$. pombe Atf1 abrogates its interaction with $\mathrm{F}$ box protein Fbh1. Curr Biol 19:1907-11.

Lawrence CL, Lawrence CL, Maekawa H, et al. (2007). Regulation of Schizosaccharomyces pombe Atf1 protein levels by Sty1-mediated phosphorylation and heterodimerization with Pcr1. J Biol Chem 282:5160-70.

Lee K, Du C, Horn M, Rabinow L. (1996). Activity and autophosphorylation of LAMMER protein kinases. J Biol Chem 271:27299303.

Lopez-Aviles S, Lopez-Aviles S, Grande M, et al. (2005). Inactivation of the Cdc25 phosphatase by the stress-activated Srk1 kinase in fission yeast. Mol Cell 17:49-59.

Lopez-Maury L, Marguerat S, Bahler J. (2008). Tuning gene expression to changing environments: from rapid responses to evolutionary adaptation. Nature reviews. Genetics 9:583-93.

Lucau-Danila A, Lelandais G, Kozovska Z, et al. (2005). Early expression of yeast genes affected by chemical stress. Mol Cell Biol $25: 1860-8$

Madrid M, Nunez A, Soto T, et al. (2007). Stress-activated protein kinase-mediated down-regulation of the cell integrity pathway mitogen-activated protein kinase Pmk1p by protein phosphatases. Mol Biol Cell 18:4405-19.

Maeda T, Wurgler-Murphy SM, Saito H. (1994). A two-component system that regulates an osmosensing MAP kinase cascade in yeast. Nature 369:242-5.

Marshall CJ. (1994). MAP kinase kinase kinase, MAP kinase kinase and MAP kinase. Curr Opin Genetics Dev 4:82-9.

Martin V, Rodriguez-Gabriel MA, McDonald WH, et al. (2006). Cip1 and Cip2 are novel RNA-recognition-motif proteins that counteract Csx1 function during oxidative stress. Mol Biol Cell 17:1176-83.

Mascarenhas C, Edwards-Ingram LC, Zeef L, et al. (2008). Gcn4 is required for the response to peroxide stress in the yeast Saccharomyces cerevisiae. Mol Biol Cell 19:2995-3007.

Mielke K, Herdegen T. (2000). JNK and p38 stresskinases-degenerative effectors of signal-transduction-cascades in the nervous system. Prog Neurobiol 61:45-60.

Millar JB, Buck V, Wilkinson MG. (1995). Pyp1 and Pyp2 PTPases dephosphorylate an osmosensing MAP kinase controlling cell size at division in fission yeast. Genes Dev 9:2117-30.

Morigasaki S, Shimada K, Ikner A, et al. (2008). Glycolytic enzyme GAPDH promotes peroxide stress signaling through multistep phosphorelay to a MAPK cascade. Mol Cell 30:108-13.

Moskvina E, Imre EM, Ruis H. (1999). Stress factors acting at the level of the plasma membrane induce transcription via the stress response element (STRE) of the yeast Saccharomyces cerevisiae. Mol Microbiol 32:1263-72.

Moye-Rowley WS, Harshman KD, Parker CS. (1989). Yeast YAP1 encodes a novel form of the jun family of transcriptional activator proteins. Genes Dev 3:283-92.

Nagahashi S, Mio T, Ono N, et al. (1998). Isolation of CaSLN1 and CaNIK1, the genes for osmosensing histidine kinase homologues, from the pathogenic fungus Candida albicans. Microbiology $144: 425-32$. 
Nebreda AR, Porras A. (2000). p38 MAP kinases: beyond the stress response. Trends Biochem Sci 25:257-60.

Neininger A, Kontoyiannis D, Kotlyarov A, et al. (2002). MK2 targets AU-rich elements and regulates biosynthesis of tumor necrosis factor and interleukin-6 independently at different post-transcriptional levels. J Biol Chem 277:3065-8.

Nguyen AN, Shiozaki K. (1999). Heat-shock-induced activation of stress MAP kinase is regulated by threonine- and tyrosine-specific phosphatases. Genes Dev 13:1653-63.

Nguyen AN, Ikner AD, Shiozaki M, et al. (2002). Cytoplasmic localization of Wis1 MAPKK by nuclear export signal is important for nuclear targeting of Spc1/Sty1 MAPK in fission yeast. Mol Biol Cell 13:2651-63.

Nguyen AN, Lee A, Place W, Shiozaki K. (2000). Multistep phosphorelay proteins transmit oxidative stress signals to the fission yeast stress-activated protein kinase. Mol Biol Cell 11:1169-81.

Nunez A, Franco A, Soto T, et al. (2010). Fission yeast receptor of activated C kinase (RACK1) ortholog $\mathrm{Cpc} 2$ regulates mitotic commitment through Wee1 kinase. J Biol Chem 285:41366-73.

Nunez A, Franco A, Madrid M, et al. (2009). Role for RACK1 orthologue $\mathrm{Cpc} 2$ in the modulation of stress response in fission yeast. Mol Biol Cell 20:3996-4009.

Ohmiya R, Kato C, Yamada H, et al. (1999). A fission yeast gene (prr1 $(+))$ that encodes a response regulator implicated in oxidative stress response. J Biochem 125:1061-6.

Ohmiya R, Yamada H, Kato C, et al. (2000). The Prr1 response regulator is essential for transcription of ste $11+$ and for sexual development in fission yeast. Mol Gen Genet 264:441-51.

Pancaldi V, Bahler J. (2011). In silico characterization and prediction of global protein-mRNA interactions in yeast. Nucleic Acids Res 39:5826-36.

Pancaldi V, Sarag OS, Rallis C, et al. (2012). Predicting the fission yeast protein interaction network. G3 (Bethesda, Md.) 2:453-67.

Park Y-D, Kang W-H, Yang W-S, et al. (2003). LAMMER kinase homolog, Lkh1, is involved in oxidative-stress response of fission yeast. Biochem Biophys Res Commun 311:1078-83.

Paul A, Wilson S, Belham CM, et al. (1997). Stress-activated protein kinases: activation, regulation and function. Cell Signal 9:403-10.

Penkett CJ, Morris JA, Wood V, Bahler J. (2006). YOGY: a web-based, integrated database to retrieve protein orthologs and associated Gene Ontology terms. Nucleic Acids Res 34:W330-4.

Perez P, Cansado J. (2010). Cell integrity signaling and response to stress in fission yeast. Curr Protein Pept Sci 11:680-92.

Posas F, Saito H. (1998). Activation of the yeast SSK2 MAP kinase kinase kinase by the SSK1 two-component response regulator. EMBO J 17:1385-94.

Posas F, Wurgler-Murphy SM, Maeda T, et al. (1996). Yeast HOG1 MAP kinase cascade is regulated by a multistep phosphorelay mechanism in the SLN1-YPD1-SSK1 "two-component" osmosensor. Cell 86:865-75.

Quinn J, Findlay VJ, Dawson K, et al. (2002). Distinct regulatory proteins control the graded transcriptional response to increasing $\mathrm{H}(2) \mathrm{O}(2)$ levels in fission yeast Schizosaccharomyces pombe. Mol Biol Cell 13:805-16.

Quinn J, Malakasi P, Smith DA, et al. (2011). Two-component mediated peroxide sensing and signal transduction in fission yeast. Antioxid Redox Signaling 15:153-65.

Rasmussen C, Rasmussen G. (1994). Inhibition of G2/M progression in Schizosaccharomyces pombe by a mutant calmodulin kinase II with constitutive activity. Mol Biol Cell 5:785-95.

Reiter W, Watt S, Dawson K, et al. (2008). Fission Yeast MAP Kinase Sty1 Is Recruited to Stress-induced Genes. J Biol Chem 283:9945-56.

Rhee SG. (2006). Cell Signaling: H2O2, a Necessary Evil for Cell Signaling. Science 312:1882-3.

Robbins DJ, Zhen E, Owaki H, et al. (1993). Regulation and properties of extracellular signal-regulated protein kinases 1 and 2 in vitro. J Biol Chem 268:5097-106.

Rodriguez-Gabriel MA, Russell P. (2008). Control of mRNA stability by SAPKs. Topics in current genetics 20:159-70.

Rodriguez-Gabriel MA, Burns G, McDonald WH, et al. (2003). RNAbinding protein Csx1 mediates global control of gene expression in response to oxidative stress. EMBO J 22:6256-66.
Rodriguez-Gabriel MA, Watt S, Bahler J, Russell P. (2006). Upf1, an RNA helicase required for nonsense-mediated mRNA decay, modulates the transcriptional response to oxidative stress in fission yeast. Mol Cell Biol 26:6347-56.

Samejima I, Mackie S, Fantes PA. (1997). Multiple modes of activation of the stress-responsive MAP kinase pathway in fission yeast. EMBO J 16:6162-70.

San Jose C, Monge RA, Perez-Diaz R, et al. (1996). The mitogenactivated protein kinase homolog HOG1 gene controls glycerol accumulation in the pathogenic fungus Candida albicans. J Bacteriol 178:5850-2.

Sanchez-Piris M, Sanchez-Piris M, Posas F, et al. (2002). The serine/ threonine kinase $\mathrm{Cmk} 2$ is required for oxidative stress response in fission yeast. J Biol Chem 277:17722-7.

Sanso M, Gogol M, Ayte J, et al. (2008). Transcription factors Pcr1 and Atf1 have distinct roles in stress- and Sty1-dependent gene regulation. Eukaryotic Cell 7:826-35.

Sanso M, Vargas-Perez I, Garcia P, et al. (2011a). Nuclear roles and regulation of chromatin structure by the stress-dependent MAP kinase Sty1 of Schizosaccharomyces pombe. Mol Microbiol 82:542-54.

Sanso M, Vargas-Perez I, Quintales L, et al. (2011b). Gen5 facilitates Pol II progression, rather than recruitment to nucleosome-depleted stress promoters, in Schizosaccharomyces pombe. Nucleic Acids Res 39:6369-79.

Sha W, Martins AM, Laubenbacher R, et al. (2013). The genomewide early temporal response of Saccharomyces cerevisiae to oxidative stress induced by cumene hydroperoxide. PloS one 8:e74939.

Shieh JC, Wilkinson MG, Buck V, et al. (1997a). The Mcs4 response regulator coordinately controls the stress-activated Wak1-Wis1-Sty1 MAP kinase pathway and fission yeast cell cycle. Genes Dev 11:1008-22.

Shieh JC, Wilkinson MG, Buck V, et al. (1997b). The Mcs4 response regulator coordinately controls the stress-activated Wak1-Wis1-Sty1 MAP kinase pathway and fission yeast cell cycle. Genes Dev $11: 1008-22$.

Shiozaki K, Russell P. (1995a). Cell-cycle control linked to extracellular environment by MAP kinase pathway in fission yeast. Nature 378:739-43.

Shiozaki K, Russell P. (1995b). Counteractive roles of protein phosphatase 2C (PP2C) and a MAP kinase kinase homolog in the osmoregulation of fission yeast. EMBO J 14:492-502.

Shiozaki K, Russell P. (1996). Conjugation, meiosis, and the osmotic stress response are regulated by Spc1 kinase through Atf1 transcription factor in fission yeast. Genes Dev 10:2276-88.

Smith DA, Toone WM, Chen D, et al. (2002). The Srk1 protein kinase is a target for the Sty1 stress-activated MAPK in fission yeast. J Biol Chem 277:33411-21.

Stock AM, Robinson VL, Goudreau PN. (2000). Two-component signal transduction. Annu Rev Biochem 69:183-215.

Sun W, Wang Z, Jiang H, et al. (2011). A novel function of the mitochondrial transcription factor Mtf1 in fission yeast; Mtf1 regulates the nuclear transcription of srk1. Nucleic Acids Res 39:2690-700.

Takada H, Nishimura M, Asayama Y, et al. (2007). Atf1 is a target of the mitogen-activated protein kinase Pmk1 and regulates cell integrity in fission yeast. Mol Biol Cell 18:4794-802.

Tatebayashi K, Tanaka K, Yang H-Y, et al. (2007). Transmembrane mucins Hkr1 and Msb2 are putative osmosensors in the SHO1 branch of yeast HOG pathway. EMBO J 26:3521-33.

Taylor BL, Zhulin IB. (1999). PAS domains: internal sensors of oxygen, redox potential, and light. Microbiol Mol Biol Rev 63:479-506.

Temple MD, Perrone GG, Dawes IW. (2005). Complex cellular responses to reactive oxygen species. Trends in Cell Biology 15:319-26.

Tibbles LA, Woodgett JR. (1999). The stress-activated protein kinase pathways. Cell Mol Life Sci 55:1230-54.

Toda T, Dhut S, Superti-Furga G, et al. (1996). The fission yeast pmk1 + gene encodes a novel mitogen-activated protein kinase homolog which regulates cell integrity and functions coordinately with the protein kinase C pathway. Mol Cell Biol 16:6752-64.

Toda T, Shimanuki M, Yanagida M. (1991). Fission yeast genes that confer resistance to staurosporine encode an AP-1-like transcription factor and a protein kinase related to the mammalian ERK1/MAP2 and budding yeast FUS3 and KSS1 kinases. Genes Dev 5:60-73. 
Toledano MB, D.A.B.B.S.D.A.D. (2003). Oxidative stress responses in yeast. In: Hohmann SMW (ed.) Yeast Stress Responses. Berlin: Springer-Verlag, 305-387.

Toone WM, Kuge S, Samuels M, et al. (1998). Regulation of the fission yeast transcription factor Pap1 by oxidative stress: requirement for the nuclear export factor Crm1 (Exportin) and the stress-activated MAP kinase Sty1/Spc1. Genes Dev 12:1453-63.

Turner R, Tjian R. (1989). Leucine repeats and an adjacent DNA binding domain mediate the formation of functional cFos-cJun heterodimers. Science 243:1689-94.

Udagawa T, Nemoto N, Wilkinson CRM, et al. (2008). Int6/eIF3e promotes general translation and Atf1 abundance to modulate Sty1 MAPK-dependent stress response in fission yeast. J Biol Chem 283:22063-75.

Umeda M, Izaddoost S, Cushman I, et al. (2005). The fission yeast Schizosaccharomyces pombe has two importin-alpha proteins, Imp1p and Cut15p, which have common and unique functions in nucleocytoplasmic transport and cell cycle progression. Genetics 171:7-21.

Vandenbroucke K, Robbens S, Vandepoele K, et al. (2008). Hydrogen peroxide-induced gene expression across kingdoms: a comparative analysis. Mol Biol Evol 25:507-16.

Vargas-Perez I, Sanchez O, Kawasaki L, et al. (2007). Response regulators SrrA and SskA are central components of a phosphorelay system involved in stress signal transduction and asexual sporulation in Aspergillus nidulans. Eukaryotic Cell 6:1570-83.

Veal EA, Day AM, Morgan BA. (2007). Hydrogen peroxide sensing and signaling. Mol Cell 26:1-14.

Veal EA, Veal EA, Findlay VJ, et al. (2004). A 2-Cys peroxiredoxin regulates peroxide-induced oxidation and activation of a stressactivated MAP kinase. Mol Cell 15:129-39.

Virginia M, Appleyard CL, McPheat WL, Stark MJ. (2000). A novel "two-component" protein containing histidine kinase and response regulator domains required for sporulation in Aspergillus nidulans. Current genetics 37:364-72.

Vivancos AP, Castillo EA, Jones N, et al. (2004). Activation of the redox sensor Pap1 by hydrogen peroxide requires modulation of the intracellular oxidant concentration. Mol Microbiol 52:1427-35.
Vivancos AP, Castillo EA, Biteau B, et al. (2005). A cysteinesulfinic acid in peroxiredoxin regulates $\mathrm{H} 2 \mathrm{O} 2$-sensing by the antioxidant Pap1 pathway. Proc Natl Acad Sci USA 102:8875-80.

Wahls WP, Smith GR. (1994). A heteromeric protein that binds to a meiotic homologous recombination hot spot: correlation of binding and hot spot activity. Genes Dev 8:1693-702.

Waskiewicz AJ, Cooper JA. (1995). Mitogen and stress response pathways: MAP kinase cascades and phosphatase regulation in mammals and yeast. Curr Opinion Cell Biol 7:798-805.

Watanabe Y, Yamamoto M. (1996). Schizosaccharomyces pombe pcr1 + encodes a CREB/ATF protein involved in regulation of gene expression for sexual development. Mol Cell Biol 16:704-11.

Weeks ME, Sinclair J, Butt A, et al. (2006). A parallel proteomic and metabolomic analysis of the hydrogen peroxide- and Sty1p-dependent stress response inSchizosaccharomyces pombe. Proteomics 6:2772-96.

Wilkinson MG, Samuels M, Takeda T, et al. (1996). The Atf1 transcription factor is a target for the Sty1 stress-activated MAP kinase pathway in fission yeast. Genes Dev 10:2289-301.

Wilson-Grady JT, Villen J, Gygi SP. (2008). Phosphoproteome analysis of fission yeast. J Proteome Res 7:1088-97.

Yun B, Farkas R, Lee K, Rabinow L. (1994). The Doa locus encodes a member of a new protein kinase family and is essential for eye and embryonic development in Drosophila melanogaster. Genes Dev 8:1160-73.

Zhan K, Vattem KM, Bauer BN, et al. (2002). Phosphorylation of eukaryotic initiation factor 2 by heme-regulated inhibitor kinaserelated protein kinases in Schizosaccharomyces pombe is important for fesistance to environmental stresses. Mol Cell Biol 22:7134-46.

Zhou C, Arslan F, Wee S, et al. (2005). PCI proteins eIF3e and eIF3m define distinct translation initiation factor 3 complexes. BMC biology 3:14.

Zhou X, Ma Y, Sugiura R, et al. (2010). MAP kinase kinase kinase (MAPKKK)-dependent and -independent activation of Sty1 stress MAPK in fission yeast. J Biol Chem 285:32818-23. 